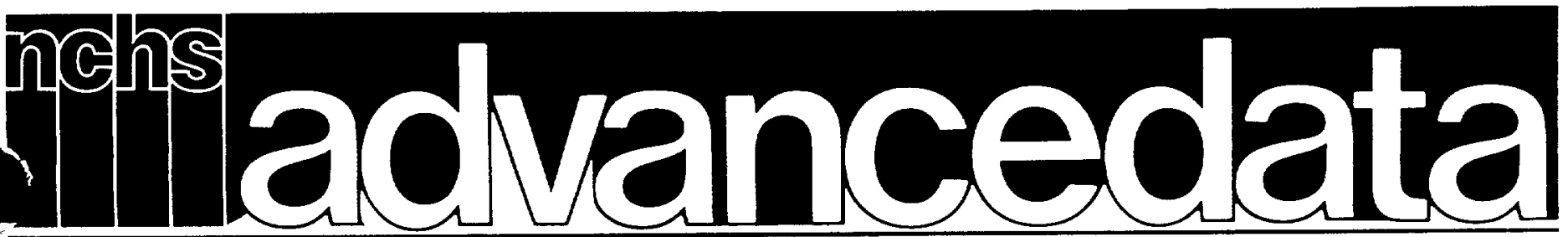

From Vital and Health Statistics of the National Center for Health Statistics

Number 86 - October 8, 1982

\title{
Drug Utilization in Office Visits to Primary Care Physicians: National Ambulatory Medical Care Survey, 1980
}

\author{
By Beulah K. Cypress, Ph.D., Division of Health Care Statistics
}

\section{Introduction}

This report presents statistics on drug utilization during office visits to general and family practitioners, internists, pediatricians, and obstetrician-gynecologists, inthe physicians generally acknowledged to be most anivolved in the delivery of primary health care. The data were gathered in 1980 by the National Center for Health Statistics by means of the National Ambulatory Medical Care Survey.

For purposes of health manpower legislation (PL94 $484,1976)$, Congress identified general and family practitioners, internists, and pediatricians as primary care providers. However, it is the policy of the American Medical Association to include obstetrician-gynecologists in the group of primary care providers. A 1978 Institute of Medicine study defined primary health care in terms of the scope, character, and integration of the services provided. 1 The report indicated that although primary care may be provided by many types of health professionals and by physicians in many different specialties, the physicians whose practice content fit the dimensions of primary care most closely were general and family practitioners, internists, pediatricians, and obstetrician-gynecologists. The National Ambulatory Medical Care Survey was the principal source of data used by the Institute of Medicine to describe the content of primary health care.

The National Ambulatory Medical Care Survey is a probability sample survey conducted annually through 1981 by the Division of Health Care Statistics of the Vational Center for Health Statistics. The technical otes at the end of this report provide brief information about the source of the data, sampling errors, and definitions of terms. A complete description of the survey including limitations and definitions was published in Vital and Health Statistics, Series 13, No. 66.2 The methodology used to collect and process the drug information is described in Vital and Health Statistics, Series 2, No. 90.3

The Patient Record form used in the 1980 survey is reproduced in figure 1 . Up to eight specific drugs, either new or continued during the visit, may be recorded by the physician in item 11 , parts $a$ and $b$. In order to present accurately what the physician ordered, prescribed, or provided, drug mentions used in this report are based on the physicians' entries on the Patient Record forms. These entries were brand or generic names of prescription $(\mathrm{R})$ or nonprescription (over-the-counter) drugs, and in some instances the physician recorded a therapeutic effect; e.g., "allergy relief."

\section{Data highlights}

\section{Visit characteristics}

Primary care physicians constituted 54 percent of the NAMCS physician universe, but had 66 percent of the office visits and accounted for 74 percent of all drug mentions (table 1). Among this group of physicians, general and family practitioners (GFP's) had a disproportionately large share of visits and drug mentions. They had 33 percent of the visits and 41 percent of the drug mentions although they represented only 23 percent of the physician universe. Obstetrician-gynecologists (OBG's) accounted for only 5 percent of the drug mentions compared with 10 percent of the visits, mainly because a relatively large proportion of their visits involve prenatal and postpartum 


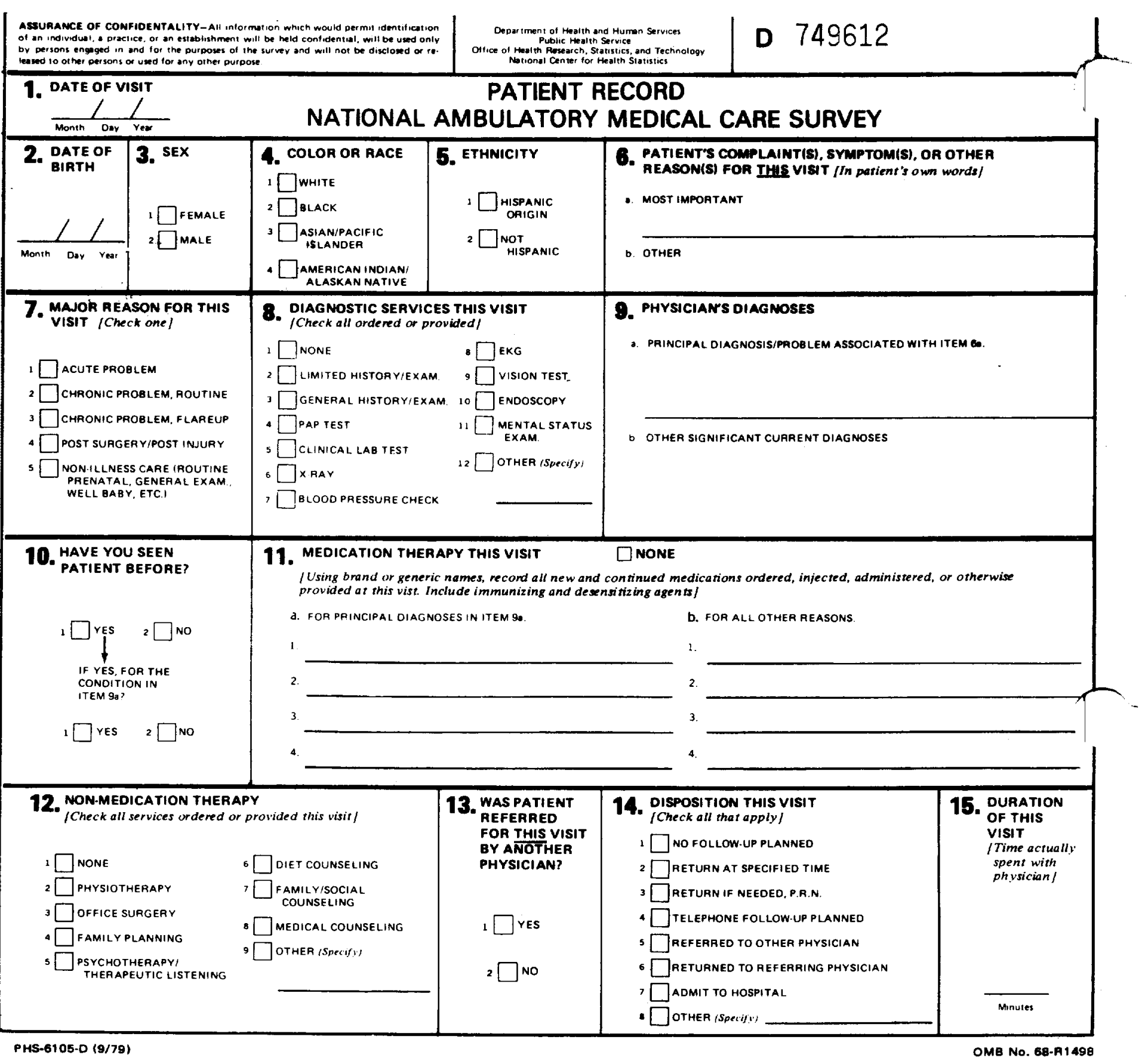

Figure 1. Patient Record from the National Ambulatory Medical Care Survey

care, and examinations for which drugs are generally not indicated.

The patterns of medication therapy presented in this report differ by specialty because drug utilization is highly related to the patient's age, sex, and condition. Although GFP's, internists, pediatricians, and OBG's provide primary care, their patients have different demographic characteristics and present more, or less, of certain diagnoses. Patterns of medical care thus vary depending on the case-mix. Table 2 shows the distribution of office visits to primary care physicians by age and sex of the patient. By age group, GFP's see a more heterogeneous group of patients than do the other physicians.
Internists provide care chiefly to adults over 24 years of age, and to a larger proportion of patients over 44 years of age (69 percent) than do GFP's ( 44 percent). Pediatricians chiefly treat children under 15 years of age. Visits by women in the child-bearing years, 15-44, account for 87 percent of the OBG's caseload. Because medication therapy, diagnosis, and the patient's age and sex are highly intercorrelated, the range of drug utilization and the classes of drugs prescribed vary among specialis

\section{Office visits and drug mentions}

The number of office visits, the number and percent of visits in which one or more drugs were prescribed (drug 
visits), and the number of drug mentions are shown in table 3. The drug mention rate is the number of drug menons divided by the number of visits; e.g., GFP's reorded an estimated 279,186,000 drug names on Patient Record forms during 191,744,000 visits, which results in a drug mention rate (DMR), or average over all visits, of 1.46 drugs per visit. Another approach to measuring drug use is to divide the number of drug mentions by the number of $d r u g$ visits (a visit in which one or more drugs were ordered). Thus, when drugs were prescribed, the average number a patient received (drug intensity rate, DIR) when visiting a GFP was 1.93 . The percent of drug visits and the DIR are used in this report to make comparisons among specialties.

Proportions of total visits with one or more drugs prescribed were similar for GFP's ( 75 percent), internists ( 76 percent), and pediatricians ( 71 percent). Only 44 percent of OBG's visits included any drugs, reflecting the large volume of visits for routine prenatal care and gynecological examinations. However, the frequency of drug visits varies by age of the patient. The rising proportions of drug visits after age group 15-24 years for GFP's and internists is shown in figure 2, in which there is a striking similarity in the configuration of the two curves.

In figure 3 proportions of drug visits to GFP's are compared with those to pediatricians. Children under 1 years of age who visited GFP's were more likely to e given at least one medication than were their counterparts who visited pediatricians. This effect was the most

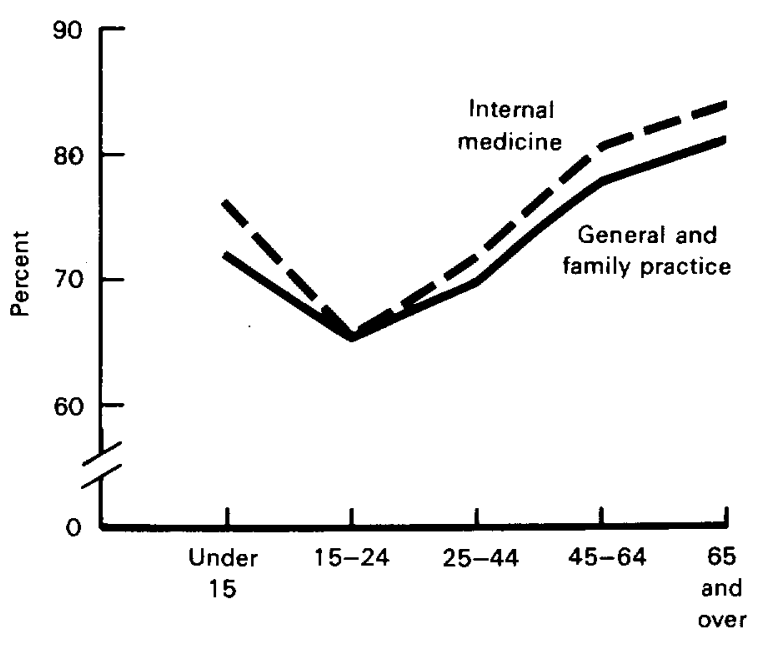

Age in years

Figure 2. Percent of drug visits by age of patient and selected primancare physician specialty: United States, 1980.

pronounced for the age group 3-5 years. Although the GFP routinely treats children in the same age range as those of the pediatrician, visits to the pediatric specialist are more likely to be for routine examinations where medication therapy is not always indicated. Two preventive health care diagnoses, health supervision of infant or child and general medical examination, accounted for 27 percent of visits to pediatricians by

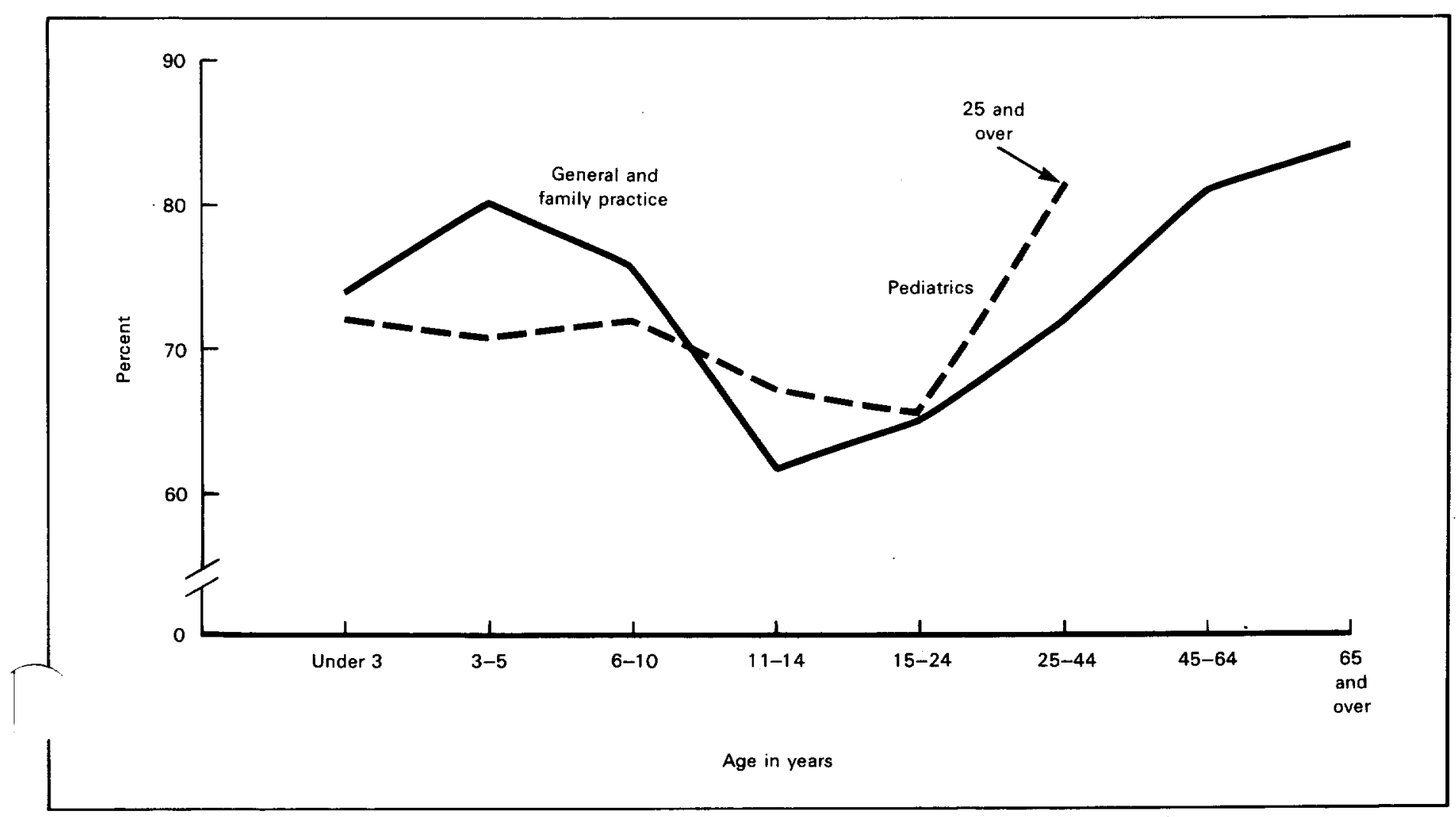

Figure 3. Percent of drug visits by age of patient and selected primary care physician specialty: United States, 1980 
children under 11 years of age compared with only 16 percent of those to GFP's.

An even more marked difference is evident in figure 4 in which proportions of drug visits by the age group of women visiting GFP's and OBG's are plotted. Percents are consistently higher for GFP's than for OBG's. The curves exhibit a similar pattern of change except that women's drug visits to GFP's decline until age group 15-24 years while the low point of drug visits to OBG's is at age group 25-44 years. The lower proportions of drug visits found in the OBG's practice is explained by the preponderance of visits with diagnoses of normal pregnancy, postpartum care, and gynecological examinations (a total of 48 percent of all visits). Only 7 percent of women's visits to GFP's were represented by these diagnoses.

On the average, the highest number of drugs prescribed during drug visits was by internists, with a rate of 2.24 drugs per drug visit, followed by GFP's with 1.93 (table 3 ). These rates are plotted by patient age group in figures 5-7. Figure 5 illustrates drug intensity rates for GFP's and internists. As expected, rates increase with increasing age after age group 15-24 years, regardless of which of the two specialists was visited. At that point, however, the curves diverge, and beginning with age group 25-44 years, internists prescribed higher numbers of medications than GFP's did.

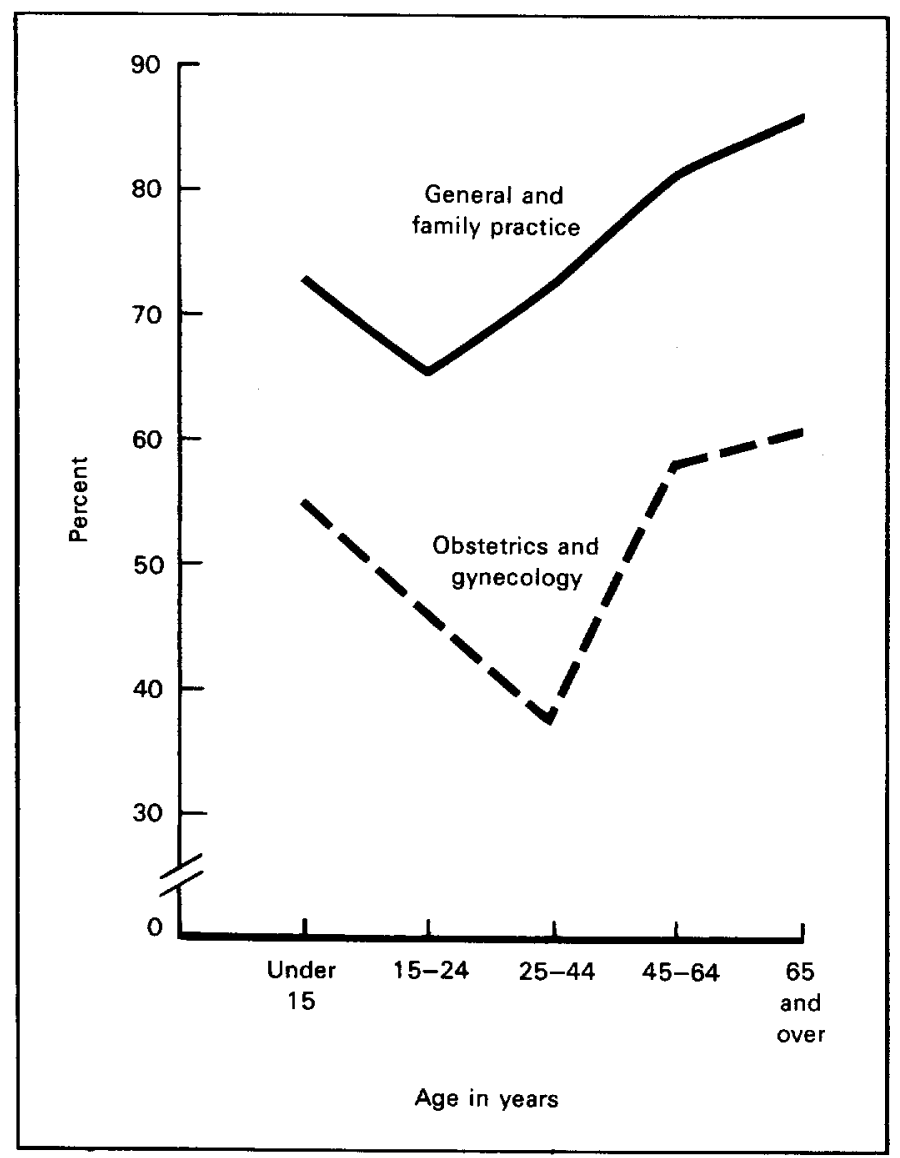

Figure 4. Percent of drug visits by age of female patient and selected primary care physician specialty: United States, 1980

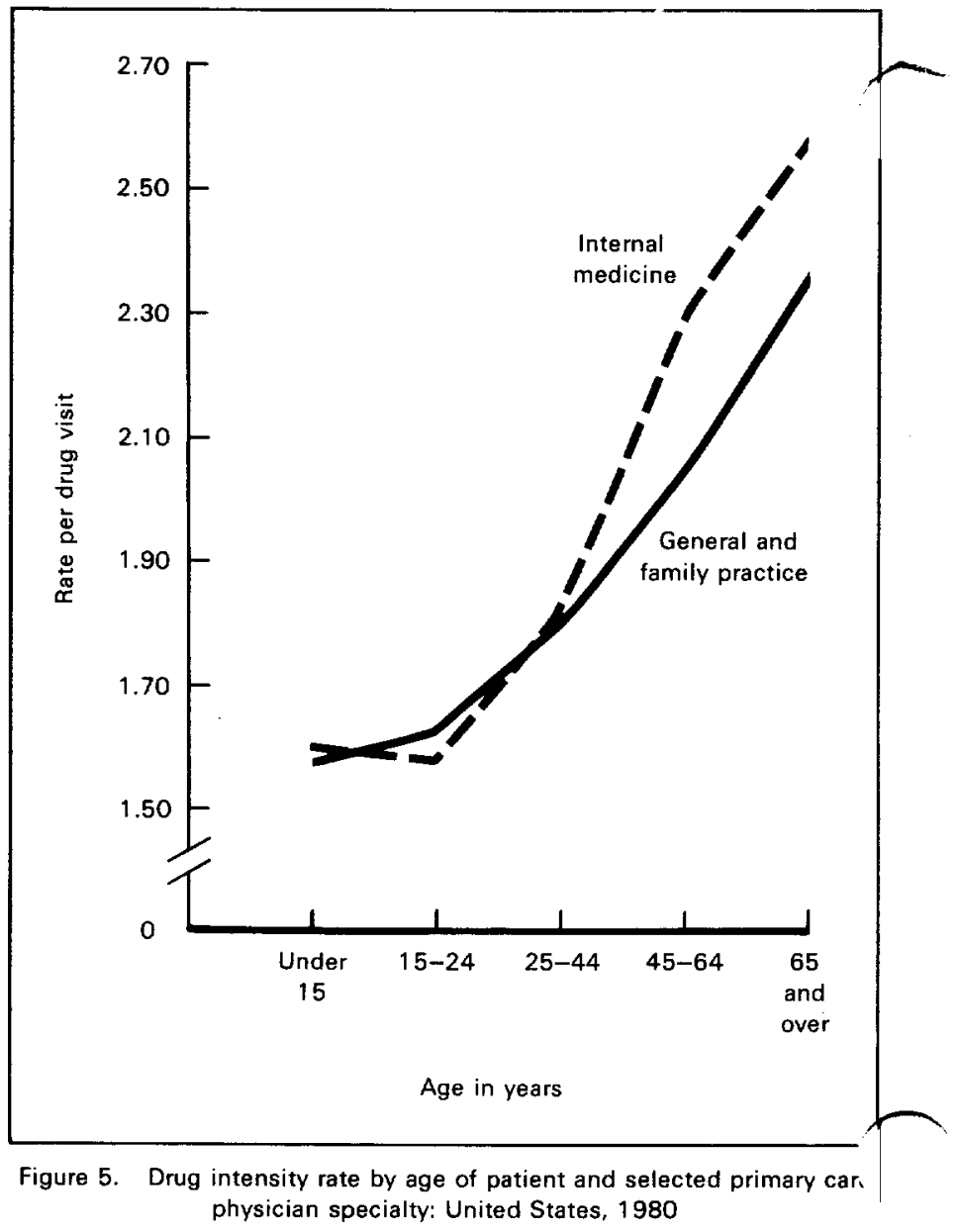

Differential diagnosis is likely to contribute to this difference in rates.

Although it was shown in figure 3 that a higher percent of visits by children under 11 years of age to GFP's included one or more drugs than did those to pediatricians, figure 6 shows that when drugs were used, the average numbers prescribed by both types of physicians were very close.

For every age group shown in figure 7, GFP's prescribed a higher average number of drugs than OBG's did, and the number prescribed tended to increase with increasing age group after 15-24 years for GFP's and after 25-44 years for OBG's. The data illustrated in figures 4 and 7 reveal that not only did GFP's have more drug visits, but they also prescribed more drugs during those visits than OBG's did. However, these findings are clearly related to the lower proportion of illness-related visits made to OBG's, as noted previously.

\section{Number of medications}

The proportions of visits that included precisel one, two, three, or four or more drugs are listed in table 4. In the first part of this table distributions are based on all visits and thus they include a "none" category. In the lower section, distributions are based on the number of 


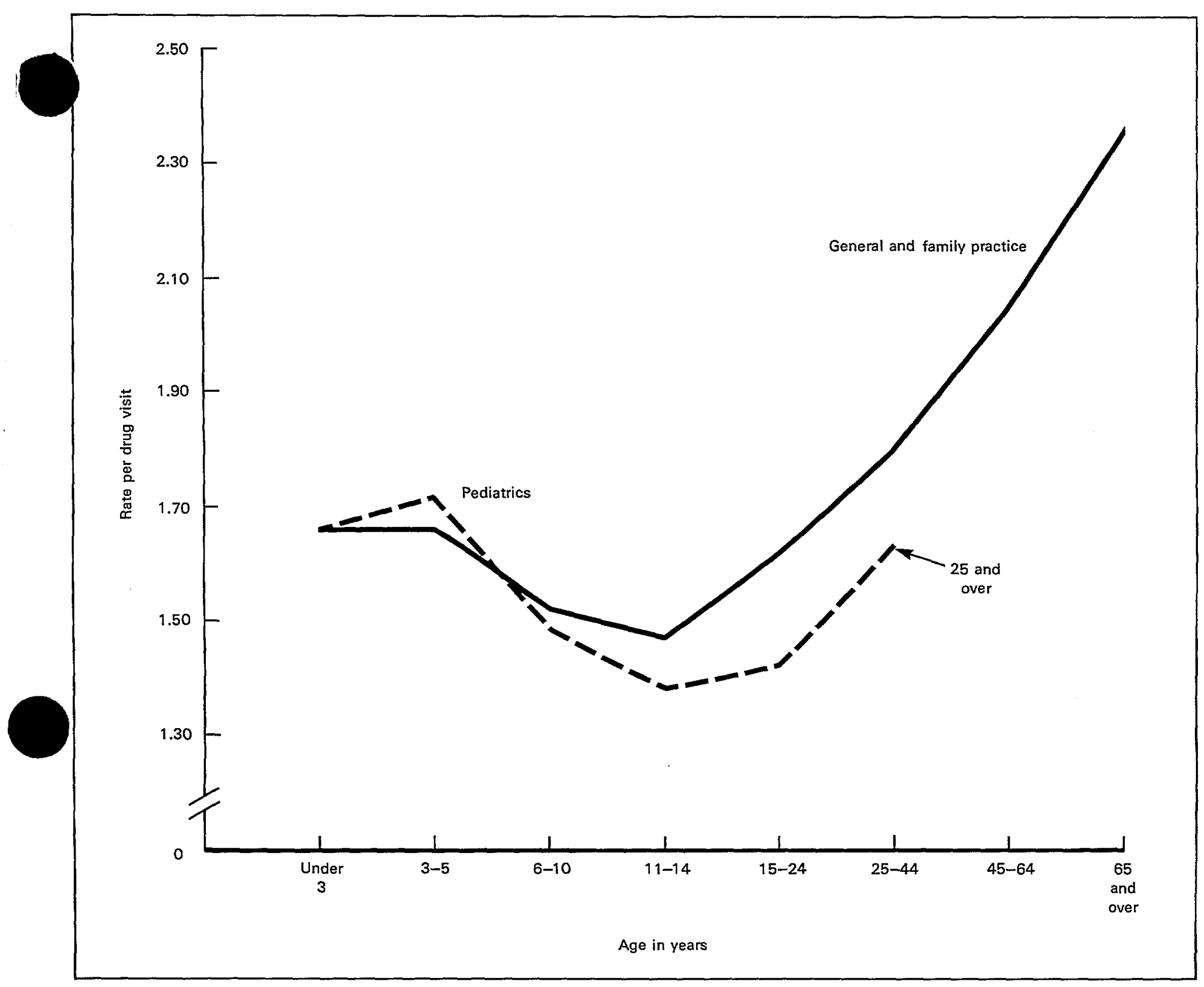

Figure 6. Drug intensity rate by age of patient and selected primary care physician specialty: United States, 1980

drug visits and, therefore, arrays do not include the "none" category. The proportions in this category are simply the complements of the percents of drug visits shown in table 3. For patients treated by GFP's, internists, and pediatricians, the largest proportions of visits were in the category of one drug mention, but the majority of visits to OBG's had no drugs mentioned. When OBG's did order drugs, 72 percent of visits included only one. Internists were more likely than other primary care physicians to order three or more drugs. About 34 percent of their drug visits included this number compared with 24 percent of GFP's, 13 percent of pediatricians, and 7 percent of OBG's. This was not unexpected in view of their relatively high proportion of visits by the elderly. It has been shown that, in general, and for certain diagnoses, the number of drugs ordered increases as the patient's age group increases. $4-6$

\section{Drug status characteristics}

NAMCS drug data are characterized by entry status (brand name, a generic entity, or therapeutic effect), prescription status (prescription or over-thecounter drug), and composition status (single ingredient, combination drug, or multivitamin). Drug mentions are distributed by these variables in table 5 . The most common method employed by physicians to enter drugs on

aInclusion of brand or trade names is for identification only and does not imply endorsement by the Public Health Service or the U.S. Department of Health and Human Services. 


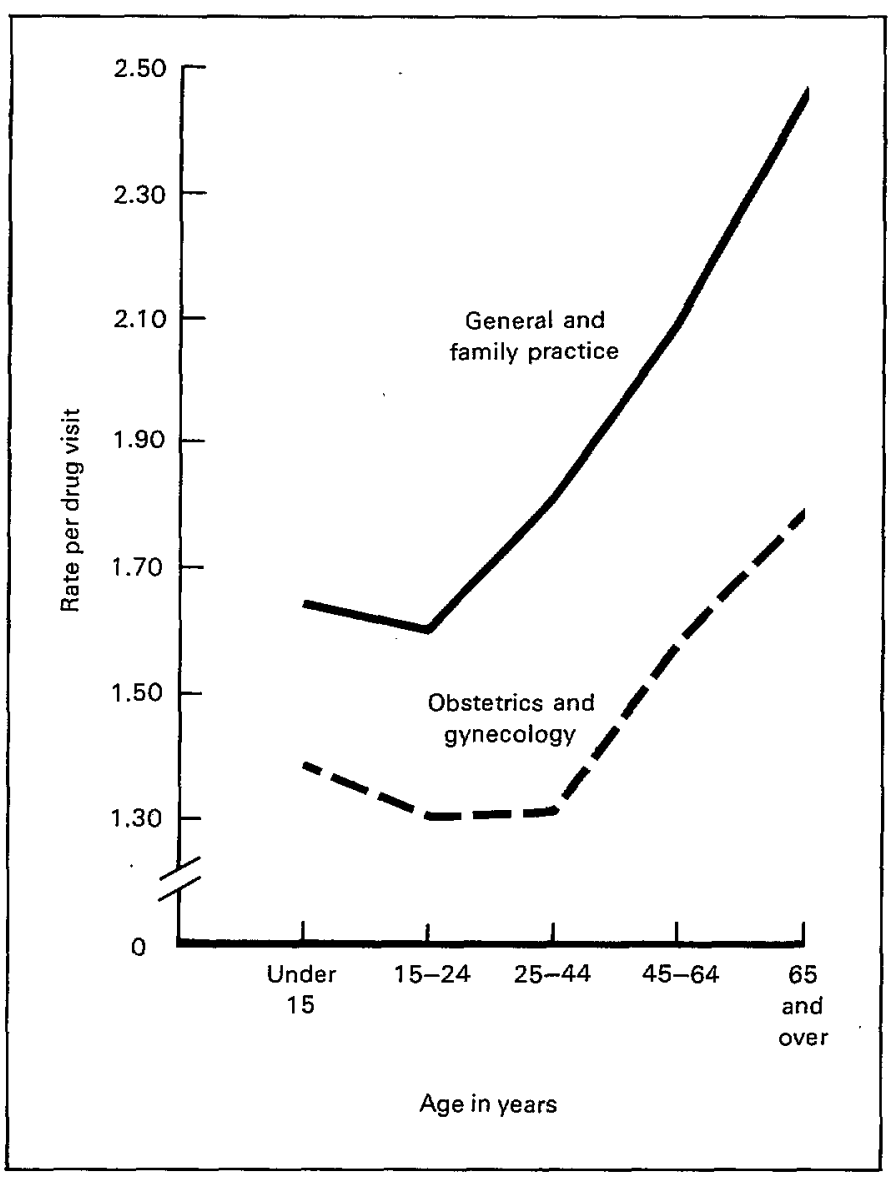

Figure 7. Drug intensity rate by age of female patient and selected primary care physician specialty: United States, 1980

the Patient Record form was by brand name. GFP's, internists, and OBG's used brand names (manufacturer's product name) in over 70 percent of mentions; pediatricians in about 58 percent. Pediatricians are more likely than other specialists to enter drugs by generic name because of their frequent use of immunizing agents and other injectable drugs. Prescription drugs were also more frequently ordered than over-the-counter drugs, ranging from 76 percent of mentions by OBG's to 85 percent of those by internists. Drugs consisting of a single principal ingredient were more likely to be prescribed than combination drugs by internists, GFP's, pediatricians, and OBG's, in declining order of frequency.

The NAMCS file also includes information on the Federal control status of each drug utilized. Drugs under the regulatory control of the Drug Enforcement Administration of the U.S. Department of Justice are assigned by them to one of five schedules based on potential for abuse and psychological or physical dependence, ranging from schedule I with the highest potential for abuse and dependence to schedule $V$ with the lowest (see reference 3 for a more detailed explanation of the schedules and examples). Drug mentions are classified in table 5 according to whether they are controlled or uncontrolled drugs. A very small proportion of drugs listed by primary care physicians were federally controlled, amounting to 11 percent of all mentions by GFP's, 8 percent by internists, and 5 percent each of those by pediatricians and OBG's. For GFP's, internists, and OBG's, the majority of controlled drugs were in schedule IV $(53,58$, and 62 percent, respectively). For pediatricians, 62 percent of controlled drugs mentioned were in schedule $\mathrm{V}$.

\section{Therapeutic categories}

Each specific drug mentioned in NAMCS is a member of a group of drugs identified by the desired therapeutic effect. These groups are based on the classification system of the American Hospital Formulary Service. ${ }^{7}$ Drug mentions are aggregated by therapeutic categories in table 6 . The leading category of drugs used varied among the primary care physicians, reflecting the demographic and clinical characteristics of their patients. For GFP's, central nervous system drugs accounted for the largest share of their mentions (19 percent). Internists used cardiovascular drugs ( 21 percent) proportionately more often than other drugs. Pediatricians most often used anti-infective agents ( 29 percent). Hormones and synthetic substitutes constituted the major portion of mentions by OBG's ( 26 percent).

The five leading categories prescribed by both GFP's and internists, although in different order of frequency, were anti-infective agents; cardiovascular drugs; central nervous system drugs; electrolytic, caloric, and water balance; and hormones and synthetic substitutes. These five categories accounted for 62 percent of drug utilization by GFP's and 70 percent of that by internists. Three of these classes were also among the five most frequently used by OBG's: anti-infective agents, central nervous system drugs, and hormones and synthetic substitutes. Two other categories frequently ordered by OBG's were skin and mucous membrane preparations (10 percent) and vitamins (19 percent). Pediatricians prescribed antihistamine drugs; expectorants and cough preparations; and serums, toxoids and vaccines proportionately more frequently than did the other primary care physicians.

\section{Specific drug mentions}

Because GFP's see a large number of patients in every age group ranging from infants to the elderly, the number and percent of the most frequently ordered specific drugs are ranked by age group in table 7 . The other primary care specialties have a more homogeneous patient load. Therefore, specific drugs are listed but not shown by age group, for internists (chiefly adults) in table 8 , for pediatricians (chiefly children) in table 9 , and for OBG's (chiefly women 15-44 years of age) in table 10 . The reader is cautioned that estimates may not differ from other near estimates due to sampling variability. Therefore, ranks may be somewhat artificial.

To treat patients under 15 years of age with medica- 
tion therapy, GFP's prescribed Ampicillin, Amoxicillin, and Penicillin in a total of 11 percent of drug mentions. Dimetapp was the leading antihistamine ordered (4 percent). Diphtheria and tetanus toxoids and pertussis vaccine (DPT), and poliomyelitis vaccine each accounted for 4 percent of mentions. Aspirin was mentioned in 3 percent.

Penicillin and Ampicillin led the list of drugs mentioned when patients visiting GFP's were 15-24 years old or 25-44 years old. However, the variety of drugs ordered changed perceptibly beginning with age group 25-44 years. While 16 drugs accounted for 42 percent of mentions for patients under 15 years, it took twice as many or more to account for about the same proportion of mentions for patients in the three older groups. The diuretic, Lasix, appears for the first time among the leading drugs ordered for patients 25-44 years of age. Over 1 million mentions of chorionic gonadotropin, a hormone frequently associated with a diagnosis of obesity, also appear on the list for 25-44 year old patients as well as three anorexients used in the treatment of obesity: Ionamin, Fastin, and Phentermine (all three are the generic entity phentermine, making a total of 1.7 million mentions of this substance).

An increase in the number of different diuretics utilized in treating patients $45-64$ years old reflects the increase of cardiovascular problems. Dyazide, Lasix, Hydrodiuril, Hygroton, and Hydrochlorothiazide were among the top 10 drugs ordered for this age group. Inderal, a drug often used to treat hypertension and certain heart conditions, was the second leading number of mentions. Inderal, as well as the previously mentioned diuretics were also among the most frequently mentioned drugs when patients were in the 65 years and over age group. Also frequently prescribed for this age group were Lanoxin and Digoxin, cardiovascular drugs both in the generic class digoxin; Aldomet, a hypotensive agent; and Motrin, which is commonly used to treat arthritis. The anti-diabetic agents Diabinese and Insulin were also among the leading medications ordered by GFP's for older patients.

The list of drugs prescribed by internists (table 8) closely resembles the lists of those used by GFP's for patients 45 years of age and over. One drug used by internists that is not among those most commonly used by GFP's is Fluorouracil, an antineoplastic agent.

Poliomyelitis vaccine accounted for 7 percent and DPT for 6 percent of all drugs mentioned by pediatricians. The tuberculin tine test was used in 5 percent. Antibiotics were prominently represented by Amoxicillin, Penicillin, Amoxil, Ampicillin, E.E.S., Bicillin, Larotid, V-Cillin, Erythromycin, and Ilosone. Many of the drugs used by pediatricians were also prominent in the section of table 7 (GFP's) showing the most frequent drugs ordered for patients under 15 years of age.

The multivitamins, Prenatal formula, Materna, Stuartnatal $1+1$, and Natalins were among the drugs ordered most frequently by OBG's. Table 10 also shows that Ortho-novum, Lo/ovral, Ovral, Demulen, and Norinyl were the most commonly prescribed oral contraceptives. Other drugs such as Premarin (estrogen), Monistat (used for candidiasis), Flagyl (used for trichomoniasis), and Sultrin (for vaginal infections) reflect the range of diagnoses made by OBG's.

These and other data on the practice characteristics of primary care physicians in 1980 and 1981 will appear in a future Vital and Health Statistics Series 13 publication. Questions regarding this report may be directed to the Ambulatory Care Statistics Branch by calling 301/436-7132.

\section{References}

1National Academy of Sciences, Institute of Medicine: $A$ manpower policy for primary health care. IOM Pub. No. 78-02. Washington. May 1978

2National Center for Health Statistics, R. Gagnon, J. DeLozier, and T. McLemore: The National Ambulatory Medical Care Survey, 1979 summary.Vital and Health Statistics. Series 13-No. 66. DHHS Pub. No. (PHS) 82-1727. Public Health Service. Washington. U.S. Government Printing Office. In press.

3National Center for Health Statistics, H. Koch: The collection and rocessing of drug information, National Ambulatory Medical Care Survey, United States, 1980. Vital and Health Statistics. Series 2-No. 90. DHHS Pub. No. (PHS) 82-1364. Public Health Service. Washington. U.S. Government Printing Office, Mar. 1982.

4National Center for Health Statistics, H. Koch: Drug utilization in office-practice by age and sex of the patient, National Ambulatory
Medical Care Survey, United States, 1980. Advance Data From Vital and Health Statistics, No. 81. DHHS Pub. No. (PHS) 811250. Public Health Service. Hyattsville, Md. July 26, 1982.

5National Center for Health Statistics, B. K. Cypress: Medication therapy in office visits for hypertension, National Ambulatory Medical Care Survey, 1980. Advance Data From Vital and Health Statistics, No. 80. DHHS Pub. No. (PHS) 81-1250. Public Health Service. Hyattsville, Md. July 22, 1982.

6National Center for Health Statistics, B. K. Cypress: Medication therapy in office visits for selected diagnoses, National Ambulatory Medical Care Survey, 1980. Vital and Health Statistics. Series 13No. 71. DHHS Pub. No. (PHS) 82-1732. Public Health Service. Washington. In preparation.

7 American Society of Hospital Pharmacists, Inc.: The American Hospital Formulary Service. Washington. Jan. 1980. 
Table 1. Number and percent distribution of physicians in NAMCS physician universe and percent distribution of visits and drug mentions, by physician specialty: United States, 1980

\begin{tabular}{|c|c|c|c|c|}
\hline \multirow[t]{2}{*}{ Physician specialty } & \multicolumn{2}{|c|}{ NAMCS physician universe } & \multirow[t]{2}{*}{ Visits } & \multirow{2}{*}{$\begin{array}{c}\begin{array}{c}\text { Drug } \\
\text { mentions }\end{array} \\
\text { tribution }\end{array}$} \\
\hline & Number & $\begin{array}{c}\text { Percent } \\
\text { distribution }\end{array}$ & & \\
\hline Total physicians $\ldots \ldots \ldots \ldots \ldots \ldots \ldots \ldots \ldots \ldots \ldots \ldots \ldots \ldots \ldots \ldots$ & 227,558 & 100.0 & 100.0 & 100.0 \\
\hline Total primary care physicians........ & 122,635 & 53.9 & 66.2 & 74.2 \\
\hline 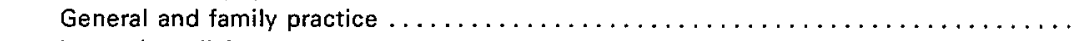 & 53,147 & 23.4 & 33.3 & 41.1 \\
\hline Internal medicine $\ldots \ldots \ldots \ldots \ldots \ldots \ldots \ldots$ & 35,199 & 15.5 & 12.1 & 17.5 \\
\hline 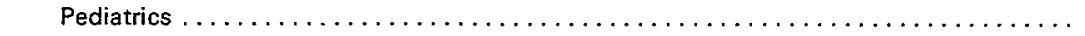 & 16,043 & 7.1 & 11.2 & 10.7 \\
\hline 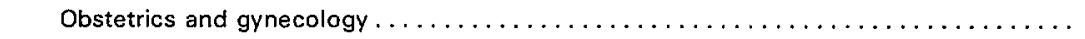 & 18,246 & 8.0 & 9.6 & 4.9 \\
\hline 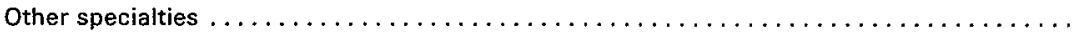 & 104,923 & 46.1 & 33.8 & 25.8 \\
\hline
\end{tabular}

Table 2. Percent distribution of visits by age and sex of patient, according to primary care physician specialty: United States, 1980

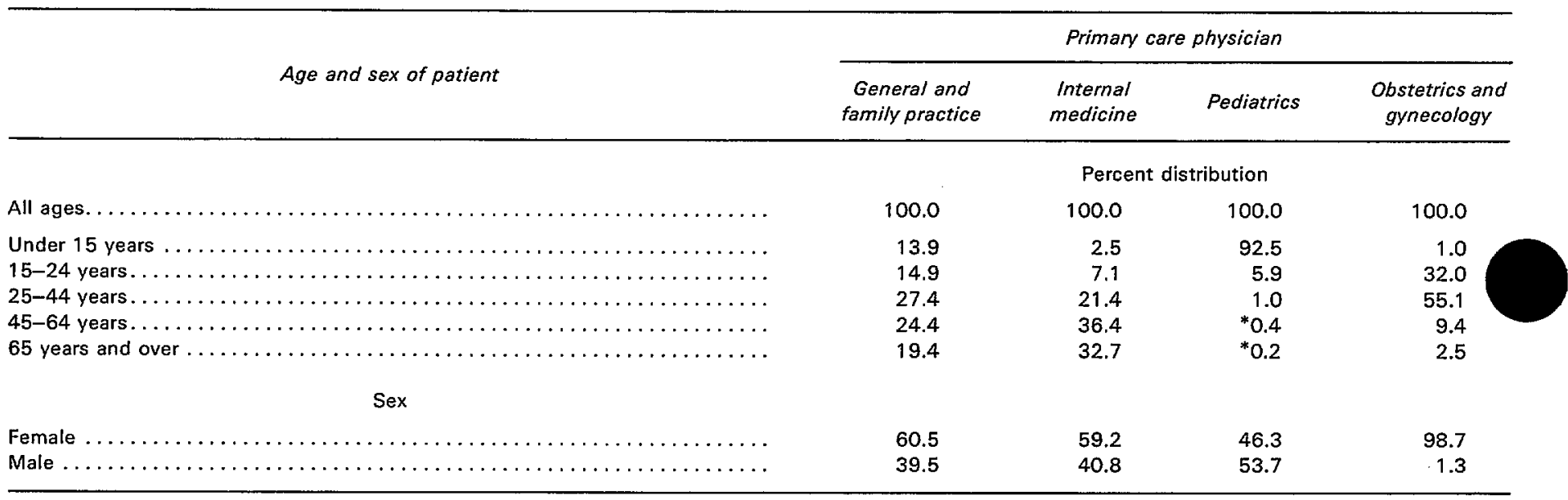

Table 3. Number of office visits, number and percent of drug visits, number of drug mentions, drug mention rate, and drug intensity rate per visit, by primary care physician specialty: United States, 1980

\begin{tabular}{|c|c|c|c|c|c|c|}
\hline Primary care physician & $\begin{array}{l}\text { All visits } \\
\text { in thousands }\end{array}$ & $\begin{array}{l}\text { Drug visits } \\
\text { in thousands }\end{array}$ & $\begin{array}{c}\text { Percent } \\
\text { drug visits }\end{array}$ & $\begin{array}{c}\text { Drug mentions } \\
\text { in thousands }\end{array}$ & $\begin{array}{c}\text { Drug mention } \\
\text { rate }^{2} \\
\text { per visit }\end{array}$ & $\begin{array}{c}\text { Drug intensity } \\
\text { rate }^{3} \\
\text { per drug visit }\end{array}$ \\
\hline General and family practice . . . . . . & 191,744 & 144,478 & 75.3 & 279,186 & 1.46 & 1.93 \\
\hline Internal medicine $\ldots \ldots \ldots \ldots \ldots$ & 69,481 & 53,091 & 76.4 & 118,943 & 1.71 & 2.24 \\
\hline Pediatrics $\ldots \ldots \ldots \ldots \ldots \ldots \ldots$ & 64,223 & 45,575 & 71.0 & 72,825 & 1.13 & 1.60 \\
\hline Obstetrics and gynecology....... & 55,123 & 23,984 & 43.5 & 33,026 & 0.50 & 1.38 \\
\hline
\end{tabular}

${ }^{1} \mathrm{~A}$ visit in which one or more drugs were prescribed.

2 Drug mentions $\div$ number of visits.

3 Drug mentions $\div$ number of drug visits. 
Table 4. Number and percent distribution of office visits by number of medications for all visits and for drug visits, according to primary care physician specialty: United States, 1980

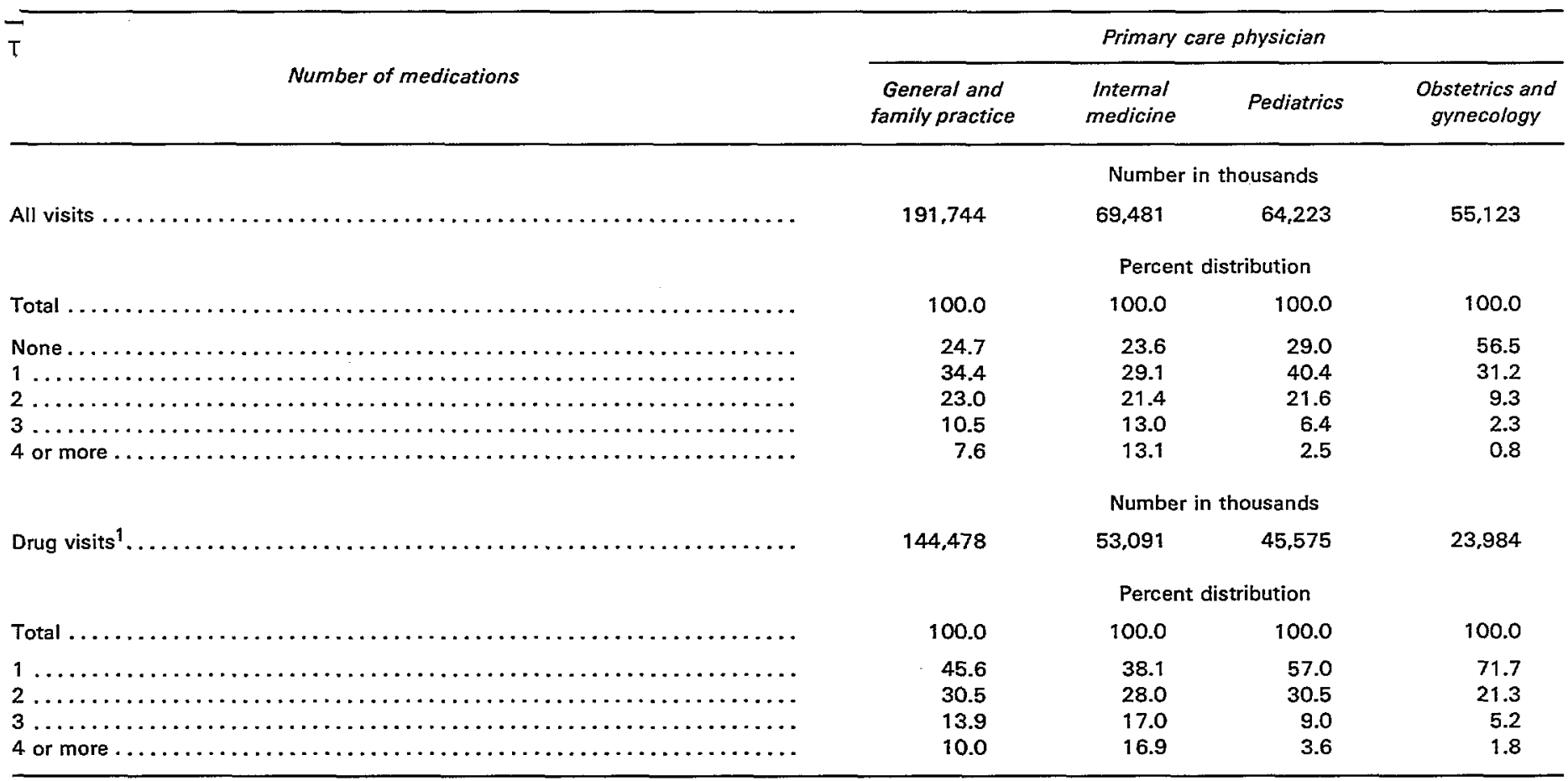

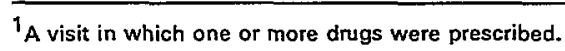

A:

$\mathrm{Te}$

Table 5. Number and percent distribution of drug mentions by selected drug status characteristics, according to primary care physician specialty: United States, 1980

Drug status characteristic

Primany care physician

Drug status characteristic

All drug mentions

\begin{tabular}{cccc}
\hline $\begin{array}{c}\text { General and } \\
\text { family practice }\end{array}$ & $\begin{array}{c}\text { internal } \\
\text { medicine }\end{array}$ & Pediatrics & $\begin{array}{c}\text { Obstetrics and } \\
\text { gynecology }\end{array}$ \\
\hline
\end{tabular}

Number in thousands

Total.

Total

$118,943 \quad 72,825$

33,026

Percent distribution

Entry status

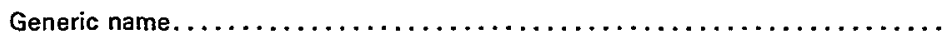

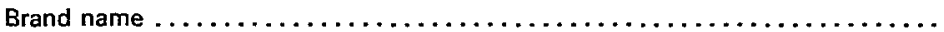

Therapeutic effect $\ldots \ldots \ldots \ldots \ldots \ldots \ldots \ldots \ldots \ldots \ldots \ldots \ldots \ldots \ldots \ldots$

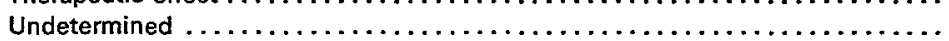

Prescription status

Prescription drug $\ldots \ldots \ldots \ldots \ldots \ldots \ldots \ldots \ldots \ldots \ldots \ldots \ldots \ldots \ldots \ldots$

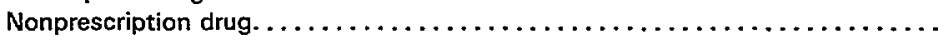

Undetermined $\ldots \ldots \ldots \ldots \ldots \ldots \ldots \ldots \ldots \ldots \ldots \ldots \ldots \ldots \ldots \ldots \ldots$

Composition status

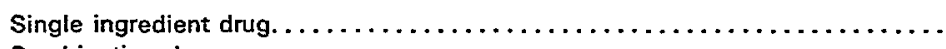

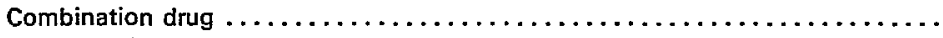

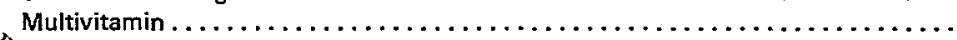

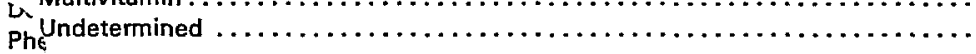

Ty!

Federal control status

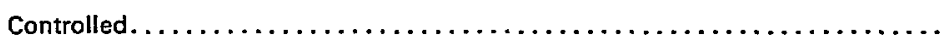

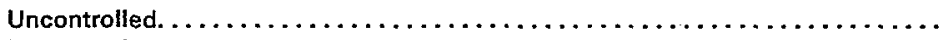

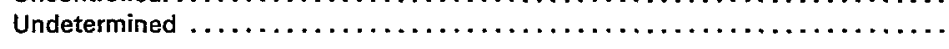

$100.0 \quad 100.0$

100.0

24.3
71.2

2.7

1.8

83.4

12.0

4.6

68.3

25.3

2.0

4.5

10.9

84.5

4.6

24.3
72.8
2.1

72.8

2.1
0.8

57.8

6.5
0.8

79.6

13.1

7.3

75.9

12.5

2.9

19.4

4.8

47.5

32.6

15.3

$\begin{array}{r}4.7 \\ \hline\end{array}$ 
Table 6. Number and percent distribution of drug mentions by therapeutic category, according to primary care physician specialty: United States, 1980

\begin{tabular}{|c|c|c|c|c|}
\hline \multirow{2}{*}{ Therapeutic category ${ }^{1}$} & \multicolumn{4}{|c|}{ Primany care physician } \\
\hline & $\begin{array}{l}\text { General and } \\
\text { family practice }\end{array}$ & $\begin{array}{l}\text { Internal } \\
\text { medicine }\end{array}$ & Pediatrics & $\begin{array}{l}\text { Obstetrics anc } \\
\text { gynecology }\end{array}$ \\
\hline \multirow[t]{2}{*}{ 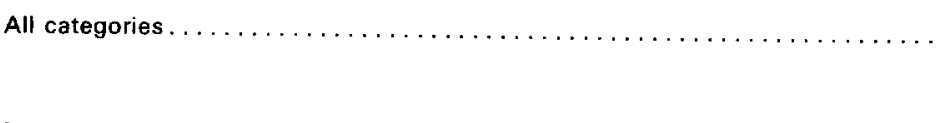 } & 279,186 & 118,943 & 72,825 & 33,026 \\
\hline & \multicolumn{4}{|c|}{ Percent distribution } \\
\hline 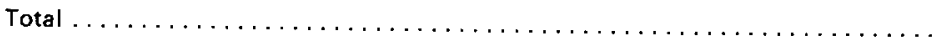 & 100.0 & 100.0 & 100.0 & 100.0 \\
\hline 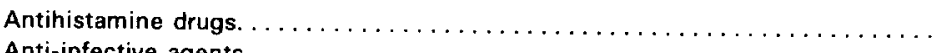 & 6.6 & 3.9 & 15.2 & 2.3 \\
\hline 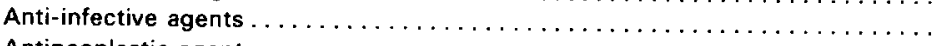 & 16.2 & 6.9 & 28.8 & 16.9 \\
\hline 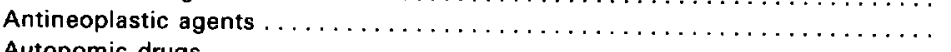 & ${ }^{*} 0.1$ & 3.4 & ${ }^{*} 0.1$ & - \\
\hline 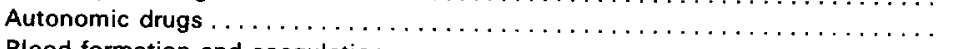 & 4.4 & 3.6 & 3.0 & $* 1.5$ \\
\hline 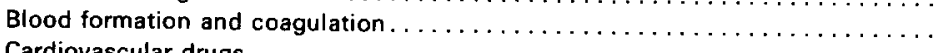 & 1.4 & 1.5 & ${ }^{*} 0.3$ & 3.5 \\
\hline 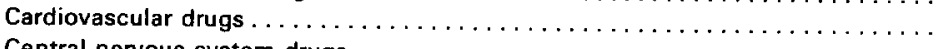 & 9.9 & 20.7 & ${ }^{*} 0.2$ & 2.5 \\
\hline 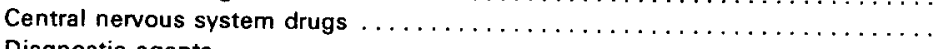 & 18.5 & 18.2 & 4.8 & 8.0 \\
\hline 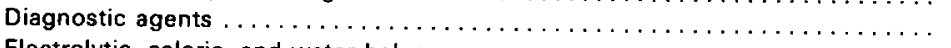 & 0.3 & ${ }^{*} 0.2$ & 4.7 & ${ }^{*} 0.1$ \\
\hline Electralytic, caloric, and water balance $\ldots \ldots \ldots \ldots \ldots \ldots \ldots \ldots \ldots \ldots$ & 9.1 & 14.3 & ${ }^{*} 0.3$ & 3.1 \\
\hline 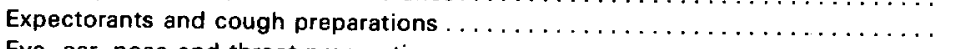 & 3.3 & 1.9 & 6.8 & ${ }^{*} 0.9$ \\
\hline Eye, ear, nose and throat preparations $\ldots \ldots \ldots \ldots \ldots \ldots \ldots \ldots \ldots \ldots \ldots \ldots \ldots$ & 1.4 & 0.8 & 3.4 & ${ }^{*} 0.5$ \\
\hline 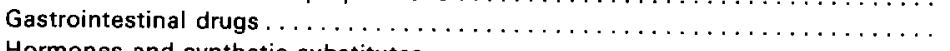 & 4.6 & 4.7 & 1.6 & $* 1.6$ \\
\hline Hormones and synthetic substitutes $\ldots \ldots \ldots \ldots \ldots \ldots \ldots \ldots \ldots \ldots \ldots$ & 8.1 & 9.7 & 1.7 & 26.4 \\
\hline 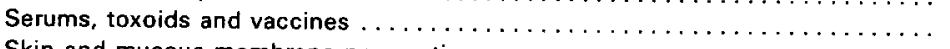 & 2.7 & 0.9 & 17.8 & ${ }^{*} 0.9$ \\
\hline 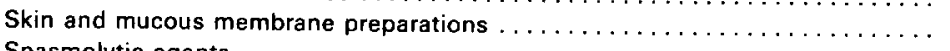 & 4.9 & 2.4 & 5.9 & 9.9 \\
\hline 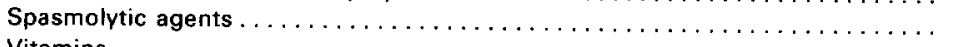 & 1.7 & 2.3 & 2.2 & ${ }^{*} 0.5$ \\
\hline 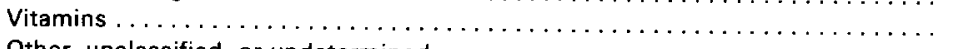 & 4.1 & 2.3 & ${ }^{*} 0.5$ & 18.6 \\
\hline Other, unclassified, or undetermined. $\ldots \ldots \ldots \ldots \ldots \ldots \ldots \ldots \ldots \ldots \ldots$ & 2.9 & 2.4 & 2.9 & 3.0 \\
\hline
\end{tabular}

${ }^{1}$ Based on the classification system of the American Hospital Formulary Service (A.H.F.S.). See reference 7. 
Table 7. Number and percent distribution of drug mentions in office visits to general and family practitioners by age of patient and most frequently named drugs: United States, 1980

\begin{tabular}{|c|c|c|c|c|c|}
\hline $\begin{array}{c}\text { Age of patient } \\
\text { and name of drug }\end{array}$ & $\begin{array}{c}\text { Number of } \\
\text { mentions } \\
\text { in } \\
\text { thousands }\end{array}$ & $\begin{array}{c}\text { Percent } \\
\text { distribution }\end{array}$ & $\begin{array}{c}\text { Age of patient } \\
\text { and name of drug } 1\end{array}$ & $\begin{array}{c}\text { Number of } \\
\text { mentions } \\
\text { in } \\
\text { thousands }\end{array}$ & $\begin{array}{c}\text { Percent } \\
\text { distribution }\end{array}$ \\
\hline Under 15 years $\ldots \ldots \ldots \ldots \ldots \ldots \ldots \ldots$ & 30,497 & 100.0 & $45-64$ years. $\ldots \ldots \ldots \ldots \ldots \ldots \ldots \ldots \ldots$ & 77.235 & 100.0 \\
\hline Ampicillin $\ldots \ldots \ldots \ldots \ldots \ldots \ldots \ldots$ & 1,326 & 4.3 & Dyazide. & 1,421 & 1.8 \\
\hline Poliomyelitis vaccine $\ldots \ldots \ldots \ldots \ldots \ldots$ & 1,272 & 4.2 & 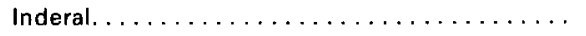 & 1,324 & 1.7 \\
\hline Dimetapp . . . . . . . . . . . . . . & 1,225 & 4.0 & 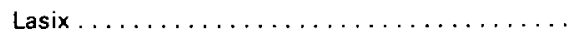 & 1,291 & 1.7 \\
\hline Diphtheria and tetanus toxoids and & & & Vitamin $\mathrm{B}-12 \ldots \ldots \ldots \ldots \ldots \ldots \ldots$ & 1,287 & 1.7 \\
\hline pertussis vaccine $\ldots \ldots \ldots \ldots \ldots \ldots$ & 1.170 & 3.8 & Motrin . . . . . . . . . . . . . & 1,125 & 1.5 \\
\hline Amoxicillin $\ldots \ldots \ldots \ldots \ldots \ldots \ldots$ & 1,095 & 3.6 & Hydrodiuril (hydrochlorothiazide) $\ldots \ldots \ldots \ldots$ & 1,026 & 1.3 \\
\hline Aspirin $\ldots \ldots \ldots \ldots \ldots \ldots \ldots \ldots$ & 995 & 3.3 & 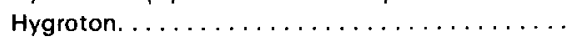 & 971 & 1.3 \\
\hline 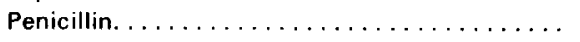 & 963 & 3.2 & Ampicillin $\ldots \ldots \ldots \ldots \ldots \ldots \ldots \ldots$ & 960 & 1.2 \\
\hline Allergy relief or shots $\ldots \ldots \ldots \ldots \ldots \ldots$ & 809 & 2.7 & Penicillin. . . . . . . . . . . . . . & 956 & 1.2 \\
\hline Keflex $\ldots \ldots \ldots \ldots \ldots \ldots \ldots \ldots$ & 572 & 1.9 & Hydrochlorothiazide $\ldots \ldots \ldots \ldots \ldots \ldots \ldots$ & 953 & 1.2 \\
\hline Erythromycin $\ldots \ldots \ldots \ldots \ldots \ldots \ldots \ldots$ & 548 & 1.8 & 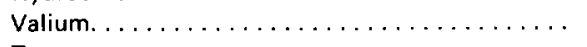 & 877 & 1.1 \\
\hline$\ldots \ldots \ldots \ldots \ldots \ldots \ldots \ldots \ldots$ & 539 & 1.8 & Tagamet $\ldots \ldots \ldots \ldots \ldots \ldots \ldots \ldots$ & 867 & 1.1 \\
\hline Amoxil (amoxicillin) $\ldots \ldots \ldots \ldots \ldots \ldots \ldots$ & 494 & 1.6 & Thyroid $\ldots \ldots \ldots \ldots \ldots \ldots \ldots \ldots$ & 849 & 1.1 \\
\hline E.E.S. (erythromycin) $\ldots \ldots \ldots \ldots \ldots \ldots$ & 490 & 1.6 & Tetracycline $\ldots \ldots \ldots \ldots \ldots \ldots \ldots \ldots$ & 759 & 1.0 \\
\hline Benadryl $\ldots \ldots \ldots \ldots \ldots \ldots \ldots \ldots$ & 484 & 1.6 & 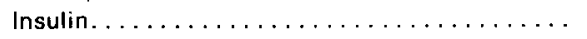 & 754 & 1.0 \\
\hline Phenergan $\ldots \ldots \ldots \ldots \ldots \ldots \ldots \ldots \ldots \ldots$ & 456 & 1.5 & Prednisone $\ldots \ldots \ldots \ldots \ldots \ldots \ldots \ldots \ldots$ & 732 & 0.9 \\
\hline E-mycin (erythromycin) $\ldots \ldots \ldots \ldots \ldots \ldots$ & 413 & 1.4 & Diuril $\ldots \ldots \ldots \ldots \ldots \ldots \ldots \ldots \ldots$ & 686 & 0.9 \\
\hline \multirow[t]{2}{*}{ Residual $\ldots \ldots \ldots \ldots \ldots \ldots \ldots \ldots$} & 17,646 & 57.7 & Lanoxin (digoxin) $\ldots \ldots \ldots \ldots \ldots \ldots \ldots$ & 677 & 0.9 \\
\hline & & & Aldomet $\ldots \ldots \ldots \ldots \ldots \ldots \ldots \ldots$ & 675 & 0.9 \\
\hline$\ldots \ldots \ldots \ldots \ldots$ & 29,989 & 100.0 & Indocin $\ldots \ldots \ldots \ldots \ldots \ldots \ldots \ldots$ & 633 & 0.8 \\
\hline$\ldots \ldots \ldots \ldots \ldots \ldots$ & 1,501 & 5.0 & Lopressor . . . . . . . . . . . . . . . . & 543 & 0.7 \\
\hline Ampicillin $\ldots \ldots \ldots \ldots \ldots \ldots \ldots \ldots$ & 1,189 & 4.0 & Clinoril $\ldots \ldots \ldots \ldots \ldots \ldots \ldots \ldots \ldots \ldots$ & 485 & 0.6 \\
\hline Aspirin $\ldots \ldots \ldots \ldots \ldots \ldots \ldots$ & 646 & 2.2 & Premarin.$\ldots \ldots \ldots \ldots \ldots \ldots \ldots \ldots \ldots$ & 485 & 0.6 \\
\hline Tetracycline . . . . . . . . . . . . . . & 539 & 1.8 & Aspirin $\ldots \ldots \ldots \ldots \ldots \ldots \ldots \ldots$ & 477 & 0.6 \\
\hline Actifed $\ldots \ldots \ldots \ldots \ldots \ldots \ldots$ & 482 & 1.6 & 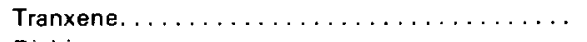 & 468 & 0.6 \\
\hline Allergy relief or shots $\ldots \ldots \ldots \ldots \ldots \ldots \ldots$ & 400 & 1.3 & Diabinese $\ldots \ldots \ldots \ldots \ldots \ldots \ldots \ldots \ldots$ & 465 & 0.6 \\
\hline 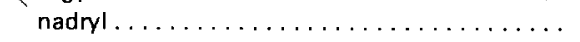 & *364 & 1.2 & Benadryl $\ldots \ldots \ldots \ldots \ldots \ldots \ldots \ldots \ldots$ & 454 & 0.6 \\
\hline ienergan expectorant with codeine . . . . . . & $* 340$ & 1.1 & Butazolidin Alka. . . . . . . . . . . . & 453 & 0.6 \\
\hline retanus toxoid $\ldots \ldots \ldots \ldots \ldots \ldots \ldots \ldots$ & $* 340$ & 1.1 & Allergy relief or shots $\ldots \ldots \ldots \ldots \ldots \ldots \ldots$ & 429 & 0.6 \\
\hline Pramet FA. $\ldots \ldots \ldots \ldots \ldots \ldots \ldots \ldots \ldots \ldots$ & ${ }^{*} 323$ & 1.1 & 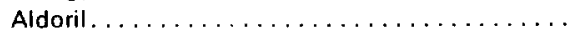 & 429 & 0.6 \\
\hline Residual $\ldots \ldots \ldots \ldots \ldots \ldots \ldots \ldots$ & 23,865 & $\begin{array}{r}1.1 \\
79.6\end{array}$ & 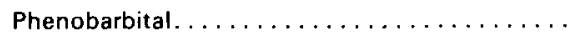 & 421 & 0.5 \\
\hline & & & E.E.S. (erythromycin) $\ldots \ldots \ldots \ldots \ldots \ldots \ldots$ & 420 & 0.5 \\
\hline$\ldots \ldots \ldots \ldots$ & 68,195 & 100.0 & 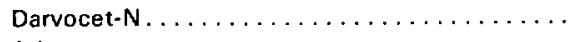 & 419 & 0.5 \\
\hline Penicillin. & 1,868 & & Ativan $\ldots \ldots \ldots \ldots \ldots \ldots \ldots \ldots \ldots$ & 408 & 0.5 \\
\hline Ampicillin $\ldots \ldots \ldots \ldots \ldots \ldots \ldots$ & $\begin{array}{l}1,000 \\
1,395\end{array}$ & 2.7 & Brethine $\ldots \ldots \ldots \ldots \ldots \ldots \ldots \ldots \ldots$ & 406 & 0.5 \\
\hline Lasix $\ldots \ldots \ldots \ldots \ldots \ldots \ldots \ldots$ & & 2.0 & Ser-ap-es $\ldots \ldots \ldots \ldots \ldots \ldots \ldots \ldots$ & 405 & 0.5 \\
\hline Tetracycline $\ldots \ldots \ldots \ldots \ldots \ldots \ldots \ldots \ldots \ldots \ldots \ldots$ & 1,327 & 1.9 & 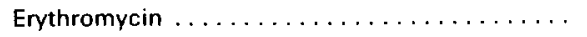 & 402 & 0.5 \\
\hline Chorionic gonadotropin $\ldots \ldots \ldots \ldots \ldots \ldots \ldots \ldots$ & 1,216 & 1.8 & $\ldots \ldots \ldots \ldots \ldots \ldots \ldots \ldots$ & 50,843 & 66.1 \\
\hline $\begin{array}{l}\text { Chorionic gonadotropin } \ldots \ldots \ldots \ldots \ldots \ldots \ldots \ldots \\
\text { Aspirin } \ldots \ldots \ldots \ldots \ldots \ldots \ldots \ldots \ldots \ldots \ldots \ldots \ldots \ldots\end{array}$ & 1.095 & 1.6 & & & \\
\hline 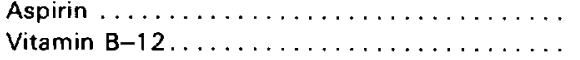 & 1,018 & 1.5 & & & \\
\hline $\begin{array}{l}\text { Vitamin } B-12 \ldots \ldots \ldots \ldots \ldots \ldots \ldots \ldots \ldots \ldots \\
\text { Allergy relief or shots } \ldots \ldots \ldots \ldots \ldots \ldots \ldots \ldots\end{array}$ & 845 & 1.2 & 65 years and over... & 73,270 & 100.0 \\
\hline 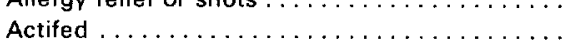 & $\begin{array}{l}837 \\
785\end{array}$ & 1.2 & $\ldots \ldots \ldots$ & 2,553 & 3.5 \\
\hline 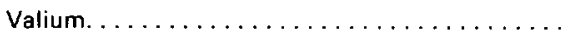 & $\begin{array}{l}785 \\
755\end{array}$ & $\begin{array}{l}1.2 \\
1.1\end{array}$ & 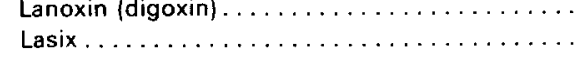 & $\begin{array}{l}2,553 \\
2,183\end{array}$ & 3.0 \\
\hline Ionamin (phentermine) $\ldots \ldots \ldots \ldots \ldots$ & 730 & 1.1 & Vitamin $\mathrm{B}-12 \ldots \ldots \ldots \ldots \ldots \ldots$ & 1.723 & 2.4 \\
\hline Hydrochlorothiazide $\ldots \ldots \ldots \ldots \ldots \ldots$ & 674 & 1.0 & 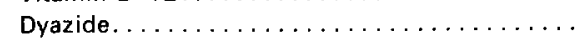 & 1,723 & 2.4 \\
\hline Tagamet $\ldots \ldots \ldots \ldots \ldots \ldots \ldots \ldots \ldots$ & 654 & 1.0 & Inderal. $\ldots \ldots \ldots \ldots \ldots \ldots \ldots \ldots$ & 1.593 & 2.2 \\
\hline Phenergan expectorant with codeine ....... & 607 & 0.9 & Aldomet $\ldots \ldots \ldots \ldots \ldots \ldots \ldots \ldots \ldots \ldots \ldots \ldots \ldots$ & 1,261 & 1.3 \\
\hline Erythromycin . . . . . . . . . . . . . . & 588 & 0.9 & Digoxin $\ldots \ldots \ldots \ldots \ldots \ldots \ldots \ldots$ & 1,168 & 1.6 \\
\hline 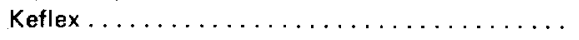 & 583 & 0.9 & 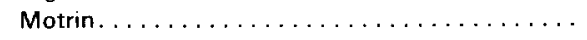 & 1,059 & 1.4 \\
\hline Thyroid $\ldots \ldots \ldots \ldots \ldots \ldots \ldots \ldots \ldots$ & 579 & 0.8 & Diabinese $\ldots \ldots \ldots \ldots \ldots \ldots \ldots \ldots$ & 972 & 1.3 \\
\hline Fastin (phentermine) $\ldots \ldots \ldots \ldots \ldots \ldots \ldots$ & 532 & 0.8 & Insulin. . . . . . . . . . . & 963 & 1.3 \\
\hline 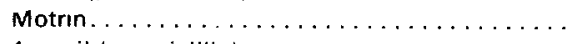 & 504 & 0.7 & Hydrodiuril (hydrochlorothiazide) . . . . . . . . & 919 & 1.3 \\
\hline Amoxil (amoxicillin) $\ldots \ldots \ldots \ldots \ldots \ldots \ldots$ & 488 & 0.7 & Hygroton. . . . . . . . . . . . . . . & 886 & 1.2 \\
\hline Phenergan $\ldots \ldots \ldots \ldots \ldots \ldots \ldots \ldots \ldots$ & 486 & 0.7 & Tagamet $\ldots \ldots \ldots \ldots \ldots \ldots \ldots \ldots \ldots$ & 790 & 1.1 \\
\hline Inderal. . . . . . . . . . . . . . . . & 476 & 0.7 & $\ldots \ldots \ldots \ldots \ldots \ldots \ldots \ldots$ & 779 & 1.1 \\
\hline Decadron $\ldots \ldots \ldots \ldots \ldots \ldots \ldots \ldots \ldots$ & 468 & 0.7 & Aldoril $\ldots \ldots \ldots \ldots \ldots \ldots \ldots \ldots \ldots \ldots \ldots \ldots \ldots \ldots \ldots \ldots$ & 778 & 1.1 \\
\hline Phentermine. . . . . . . . . . . . . & 463 & 0.7 & 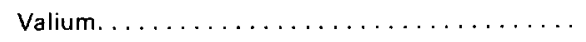 & 772 & 1.1 \\
\hline Tylenol with codeine. . . . . . . . . . . . . & 448 & 0.7 & Influenza virus vaccine, type A,B $\ldots \ldots \ldots \ldots$ & 734 & 1.0 \\
\hline Tylenol . . . . . . . . . . . . . . . . . . & 443 & 0.7 & Nitroglycerin. . . . . . . . . . . . . & 654 & 0.9 \\
\hline$\ldots \ldots \ldots \ldots \ldots$ & 438 & 0.6 & Slow-k $\ldots \ldots \ldots \ldots \ldots \ldots \ldots \ldots \ldots \ldots \ldots$ & 650 & 0.9 \\
\hline S. (erythromycin) $\ldots \ldots \ldots \ldots \ldots \ldots \ldots$ & 434 & 0.6 & isordil $\ldots \ldots \ldots \ldots \ldots \ldots \ldots \ldots \ldots$ & 639 & 0.9 \\
\hline rramycin ........ & 428 & 0.6 & Antivert. . . . . . . . . . . . . . & 622 & 0.8 \\
\hline Tranxene. . . . . . . . . . . . . . . . & 411 & 0.6 & Tetracycline $\ldots \ldots \ldots \ldots \ldots \ldots \ldots$ & 616 & 0.8 \\
\hline Residual $\ldots \ldots \ldots \ldots \ldots \ldots \ldots \ldots \ldots$ & 46,620 & 68.4 & 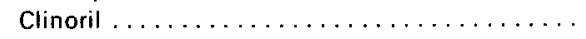 & 605 & 0.8 \\
\hline
\end{tabular}


Table 7. Number and percent distribution of drug mentions in office visits to general and family practitioners by age of patient and most frequently named drugs: United States, $1980-$ Con.

\begin{tabular}{|c|c|c|c|c|c|}
\hline $\begin{array}{c}\text { Age of patient } \\
\text { and name of drug } 1\end{array}$ & $\begin{array}{l}\text { Number of } \\
\text { mentions } \\
\text { in } \\
\text { thousands }\end{array}$ & $\begin{array}{c}\text { Percent } \\
\text { distribution }\end{array}$ & $\begin{array}{c}\text { Age of patient } \\
\text { and name of drug } 1\end{array}$ & $\begin{array}{c}\text { Number of } \\
\text { mentions } \\
\text { in } \\
\text { thousands }\end{array}$ & $\begin{array}{c}\text { Percent } \\
\text { distribution }\end{array}$ \\
\hline 65 years and over-Con. & & & 65 years and over-Con. & & \\
\hline Hydrochlorothiazide $\ldots \ldots \ldots \ldots \ldots \ldots \ldots$ & 596 & 0.8 & Lopressor . . . . . . . . . . . . . . . . & 469 & 0.6 \\
\hline Penicillin. . . . . . . $\ldots \ldots \ldots \ldots \ldots$ & 542 & 0.7 & Indocin $\ldots \ldots \ldots \ldots \ldots \ldots \ldots \ldots$ & 455 & 0.6 \\
\hline Dalmane.$\ldots \ldots \ldots \ldots \ldots \ldots \ldots$ & 522 & 0.7 & Darvocet-N . . . . . . . . . . . . . . . . & 449 & 0.6 \\
\hline Orinase $\ldots \ldots \ldots \ldots \ldots \ldots \ldots \ldots$ & 501 & 0.7 & Naprosyn . . . . . . . . . . . . . . . . & 440 & 0.6 \\
\hline Nitro-bid (nitroglycerin) $\ldots \ldots \ldots \ldots \ldots \ldots$ & 485 & 0.7 & Residual $\ldots \ldots \ldots \ldots \ldots \ldots \ldots \ldots$ & 44,171 & 60.2 \\
\hline
\end{tabular}

${ }^{1}$ Based on the physician's entry on the Patient Record form.

Table 8. Number and percent distribution of drug mentions in office visits to internists by most frequently named drugs: United States, 1980

\begin{tabular}{|c|c|c|}
\hline Name of drug ${ }^{1}$ & $\begin{array}{c}\text { Number of } \\
\text { mentions } \\
\text { in thousands }\end{array}$ & $\begin{array}{c}\text { Percent } \\
\text { distribution }\end{array}$ \\
\hline 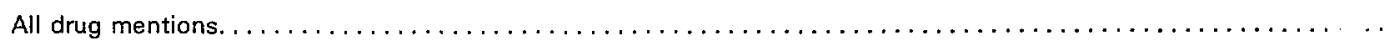 & 118,943 & 100.0 \\
\hline$\ldots \ldots \ldots \ldots \ldots \ldots \ldots$ & 4.150 & 3.5 \\
\hline 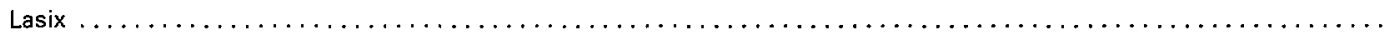 & 3,130 & 2.6 \\
\hline 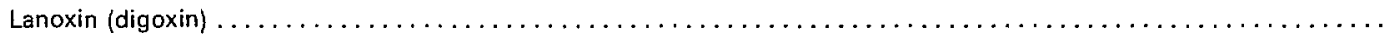 & 2,752 & 2.3 \\
\hline 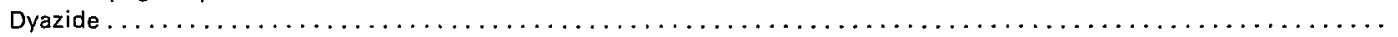 & 2,743 & $2: \therefore$ \\
\hline 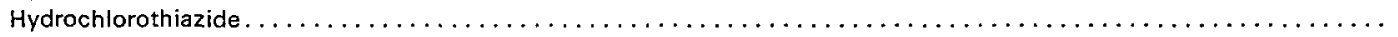 & 2,287 & 1.5 \\
\hline 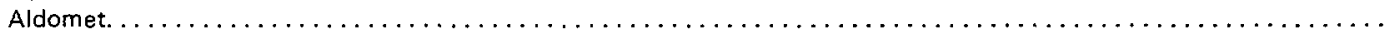 & 2,232 & 1.9 \\
\hline 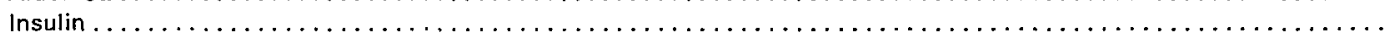 & 2,110 & 1.8 \\
\hline 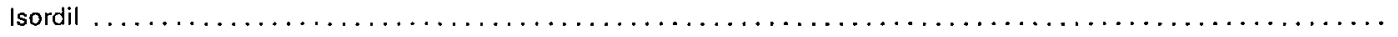 & 2,080 & 1.7 \\
\hline 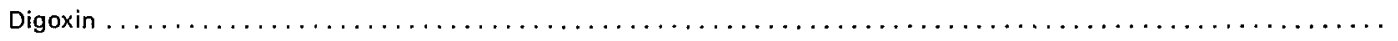 & 1,979 & 1.7 \\
\hline 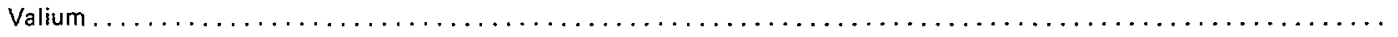 & 1,952 & 1.6 \\
\hline 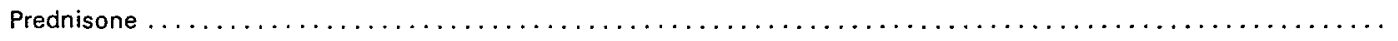 & 1,948 & 1.6 \\
\hline 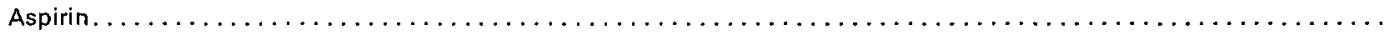 & 1,889 & 1.6 \\
\hline 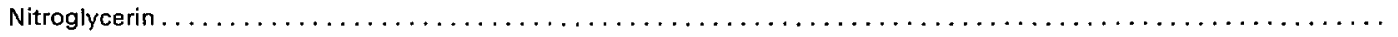 & 1.703 & 1.4 \\
\hline 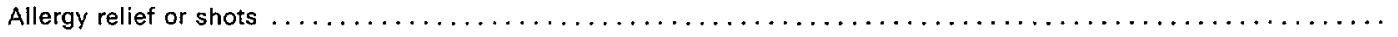 & 1,434 & 1.2 \\
\hline 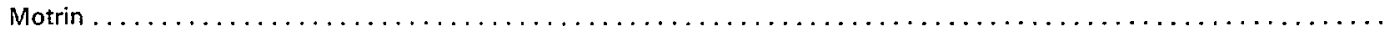 & 1,326 & 1.1 \\
\hline 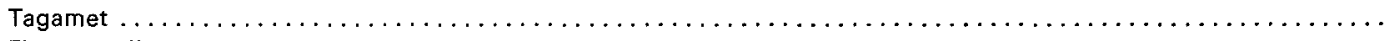 & 1,220 & 1.0 \\
\hline 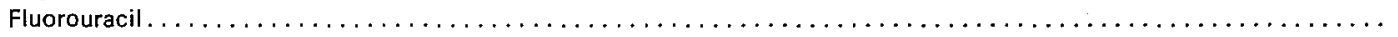 & 1,217 & 1.0 \\
\hline 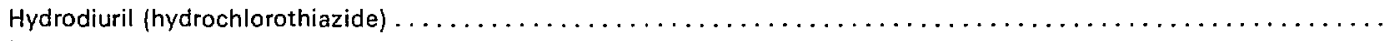 & 1,154 & 1.0 \\
\hline 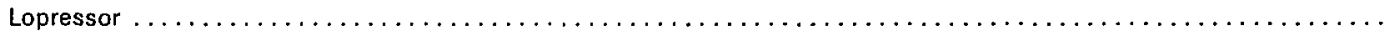 & 1,150 & 1.0 \\
\hline 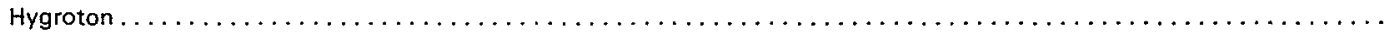 & 1,080 & 0.9 \\
\hline 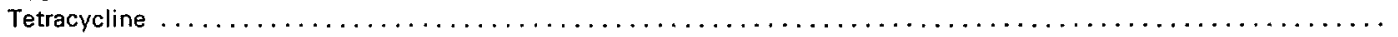 & 1,026 & 0.9 \\
\hline 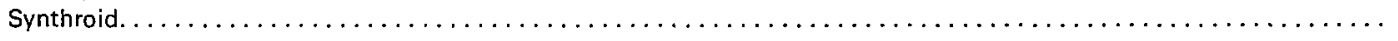 & 1,018 & 0.9 \\
\hline 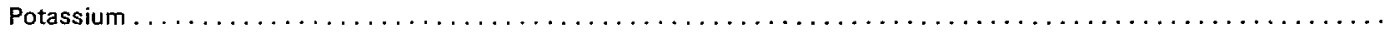 & 1,005 & 0.8 \\
\hline 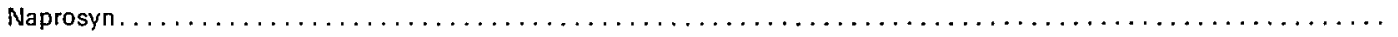 & 992 & 0.8 \\
\hline 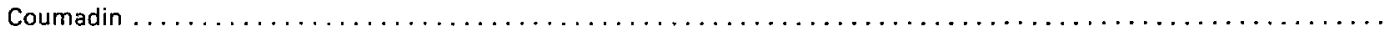 & 965 & 0.8 \\
\hline 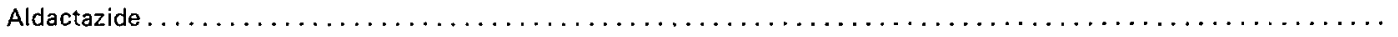 & 959 & 0.8 \\
\hline 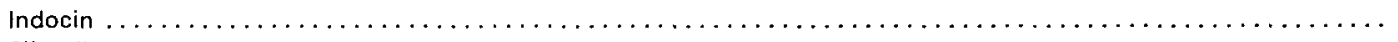 & 954 & 0.8 \\
\hline 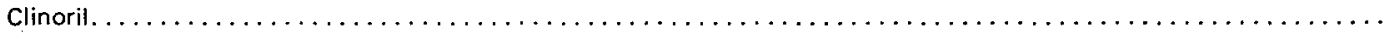 & 945 & 0.8 \\
\hline 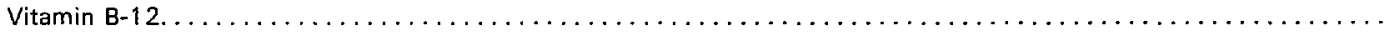 & 902 & 0.8 \\
\hline 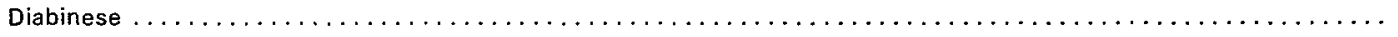 & 848 & 0.7 \\
\hline 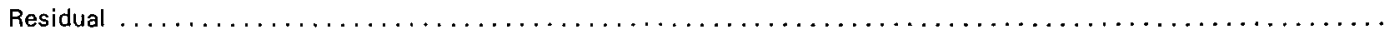 & 69,793 & 58.8 \\
\hline
\end{tabular}

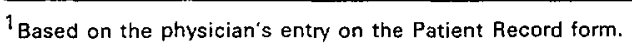


Table 9. Number and percent distribution of drug mentions in office visits to pediatricians by most frequently named drugs: United States, $198 \mathrm{n}$

Number of
mentions
in thousands

All drug mentions.

\begin{tabular}{rr}
72,825 & 100.0 \\
4,829 & 6.6 \\
4,564 & 6.3 \\
3,409 & 4.7 \\
3,146 & 4.3 \\
2,991 & 4.1 \\
2,252 & 3.1 \\
1.858 & 2.6 \\
1,538 & 2.1 \\
1.527 & 2.1 \\
1,440 & 2.0 \\
1,236 & 1.7 \\
1,078 & 1.5 \\
977 & 1.3 \\
892 & 1.2 \\
857 & 1.2 \\
809 & 1.1 \\
795 & 1.1 \\
794 & 1.1 \\
789 & 1.1 \\
753 & 1.0 \\
735 & 1.0 \\
735 & 1.0 \\
703 & 1.0 \\
656 & 0.9 \\
654 & 0.9 \\
632 & 0.9 \\
618 & 0.8 \\
593 & 0.8 \\
591 & 0.8 \\
575 & 0.8 \\
29,799 & 40.9 \\
\hline &
\end{tabular}

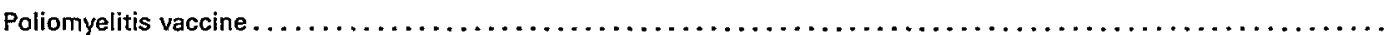

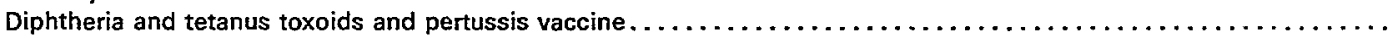

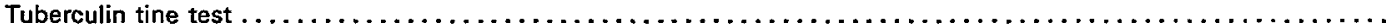

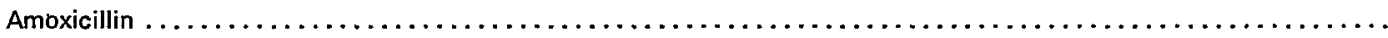

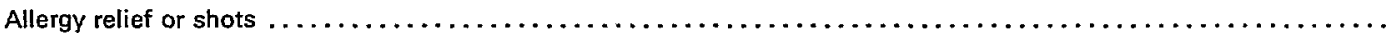

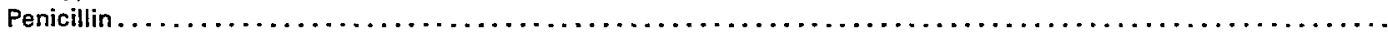

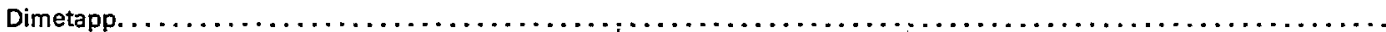

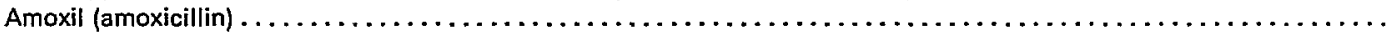

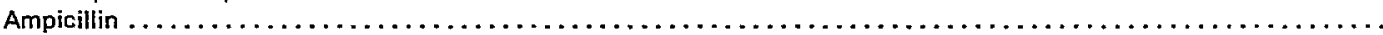

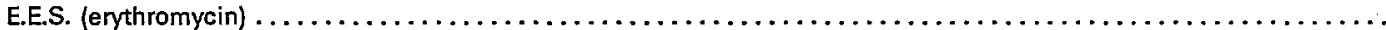

Aspirin...

Dimetane

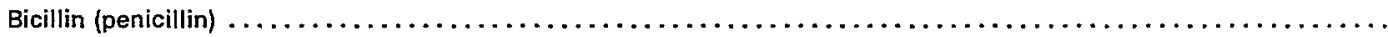

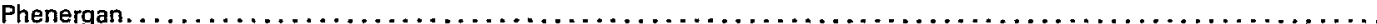

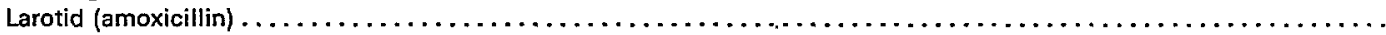

Actifed.

Vaccination (undetermined)

V-Cillin (penicillin)

Novahistine.

n.

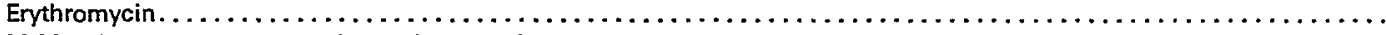

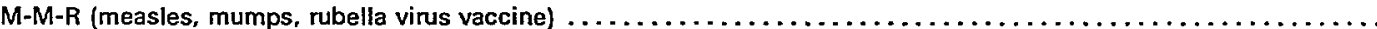

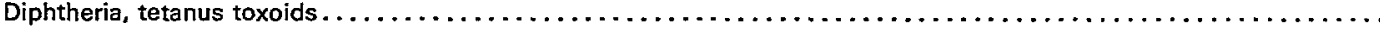

Tylenol. . .

Phenergan expectorant with codeine.

Rondec....

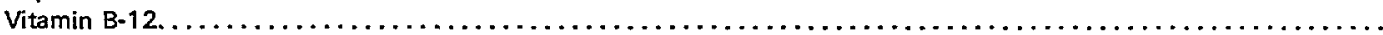

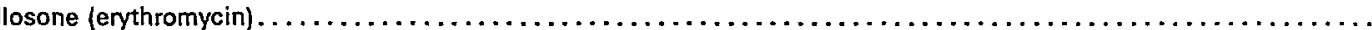

Triaminic.

Benadryl

Based on the physician's entry on the Patient Record form.

Table 10. Number and percent distribution of drug mentions in office visits to obstetrician-gynecologists by most frequently named drugs: United States, 1980

Name of drug ${ }^{1}$

Number of
mentions
in thousands

All drug mentions. . .

33,026

100.0

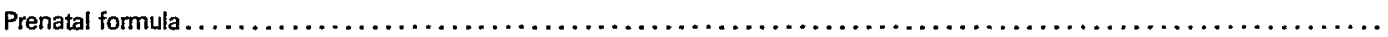

Ortho-novum.

Monistat .

Premarin .

Materna.

Lo/ovral.....

Ampicillin ....

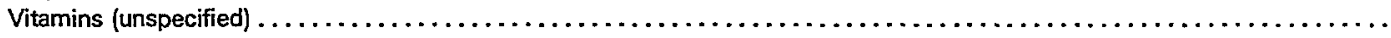

Stuartnatal $1+1$

Flagyl.... . .

Tetracycline

Ovral.

Natalins. .

Demulen.

Sultrin

Norinyl

Residual .

\footnotetext{
$1_{\text {Based on the physician's entry on the Patient Record form. }}$
} 


\section{Technical notes}

\section{Source of data and sample design}

The information presented in this report is based on data collected by the National Center for Health Statistics through its National Ambulatory Medical Care Survey (NAMCS) during 1980. The target universe of NAMCS includes office visits made within the coterminous United States by ambulatory patients to nonfederally employed physicians who are principally engaged in office practice, but not in the specialties of anesthesiology, pathology, or radiology. Telephone contacts and nonoffice visits are excluded.

NAMCS utilizes a multistage probability sample design that involves samples of primary sampling units (PSU's), physicians' practices within PSU's, and patient visits within physician practices. For 1980 a sample of 2,959 non-Federal, office-based physicians was selected from master files maintained by the American Medical Association and the American Osteopathic Association. The physician response rate for 1980 was 77.2 percent. Sampled physicians were asked to complete Patient Records (figure 1) for a systematic random sample of office visits taking place during a randomly assigned weekly reporting period. During 1980, responding physicians completed 46,081 Patient Records, on which they recorded 51,372 drug mentions. Characteristics of the physician's practice, such as primary specialty and type of practice, were obtained during an induction interview. The National Opinion Research Center, under contract to the National Center for Health Statistics, was responsible for the survey's field operations.

For a more detailed discussion of the limitations, qualifications, and definitions of the data collected in the NAMCS, see Vital and Health Statistics, Series 13. No. 66.2

Estimates presented in this report differ from the estimates reported in the National Medical Care Utilization and Expenditure Survey (NMCUES), another program of the National Center for Health Statistics (NCHS). The variation in estimates is due to differences in survey populations, data collection methodology, and definitions. The NMCUES, cosponsored by NCHS and the Health Care Financing Administration (HCFA), is a national panel survey of households in which information on visits to physicians' offices and hospital outpatient departments was collected. Preliminary survey data as well as a discussion of the survey methodology are forthcoming from NCHS and HCFA.

\section{Sampling errors and rounding of numbers}

The standard error is primarily a measure of the sampling variability that occurs by chance because

NOTE: $A$ list of references follows the text. only a sample, rather than the entire universe, is surveyed. The relative standard error of an estimate is obtained by dividing the standard error by the estimate itself and is expressed as a percent of the estimate. Relative standard errors of selected aggregate visit statistics are shown in table I. Standard errors for estimated percents of visits are shown in table II. Similar standard errors for drug statistics and percents are shown in tables III and IV. Tables I and II should be used to obtain the standard error of a specific drug mention (e.g., Dyazide). Tables III and IV should be used to obtain the standard error of a group of drug mentions (e.g., all drugs prescribed for hypertension).

Estimates of office visits have been rounded to the nearest thousand. For this reason detailed figures within tables do not always add to totals. Rates and percents were calculated on the basis of original, unrounded figures and will not necessarily agree precisely with percents calculated from rounded data.

\section{Definitions}

An ambulatory patient is an individual presenting himself for personal health services who is neither bedridden nor currently admitted to any health car institution on the premises.

A physician eligible for NAMCS is a duly licensed doctor of medicine (M.D.) or doctor of osteopathy (D.O.) currently in office-based practice who spends time in caring for ambulatory patients. Excluded from NAMCS are physicians who are hospital based; physicians who specialize in anesthesiology, pathology, or radiology; physicians who are federally employed; physicians who treat only institutionalized patients; physicians employed full time by an institution; and physicians who spend no time seeing ambulatory patients.

An office is a place that the physician identities as a

Table I. Approximate relative standard errors of estimated number of affice visits based on all physician specialties: NAMCS, 1980

\begin{tabular}{|c|c|}
\hline $\begin{array}{l}\text { Estimated number of office visits } \\
\text { in thousands }\end{array}$ & $\begin{array}{c}\text { Relative } \\
\text { standard } \\
\text { error in } \\
\text { Dercent }\end{array}$ \\
\hline 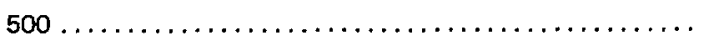 & 27.3 \\
\hline 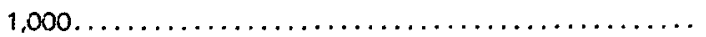 & 19.5 \\
\hline 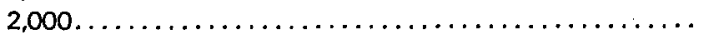 & 14.1 \\
\hline 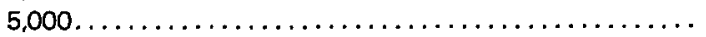 & 9.4 \\
\hline 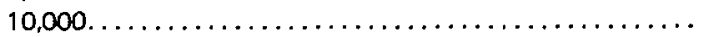 & 7.3 \\
\hline 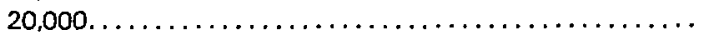 & 5.9 \\
\hline 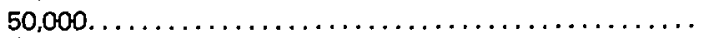 & 4.9 \\
\hline 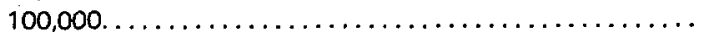 & 4.5 \\
\hline 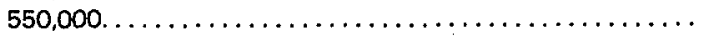 & 4.1 \\
\hline
\end{tabular}

Example of use of table: An aggregate estimate of $75,000,000$ visits has a relative standard error of 4.7 percent, or a standard error of $3,525,000$ visits $(4.7$ percent of $75,000,000$ ). 
Table II. Approximate standard errors of percents of estimated numbers of office visits based on all physician specialties: NAMCS, 1980

Base of percent

(number of office visits in thousands)

\begin{tabular}{lllllll}
\multicolumn{6}{c}{ Estimated percent } \\
\hline 1 or 99 & 5 or 95 & 10 or 90 & 20 or 80 & 30 or 70 & 50 \\
\hline
\end{tabular}

Standard error in percent

\begin{tabular}{|c|c|c|c|c|c|c|}
\hline $500 \ldots \ldots \ldots \ldots \ldots \ldots \ldots$ & 2.7 & 5.9 & 8.1 & 10.8 & 12.4 & 13.5 \\
\hline $1,000 \ldots \ldots \ldots \ldots \ldots \ldots \ldots \ldots \ldots \ldots \ldots \ldots \ldots \ldots \ldots \ldots \ldots \ldots \ldots \ldots \ldots$ & 1.9 & 4.2 & 5.7 & 7.6 & 8.7 & 9.5 \\
\hline $2,000 \ldots \ldots \ldots \ldots \ldots \ldots \ldots \ldots \ldots \ldots \ldots \ldots \ldots \ldots \ldots \ldots \ldots$ & 1.3 & 2.9 & 4.0 & 5.4 & 6.2 & 6.7 \\
\hline 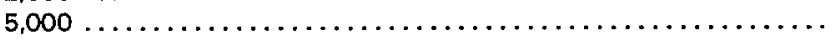 & 0.8 & 1.9 & 2.6 & 3.4 & 3.9 & 4.3 \\
\hline 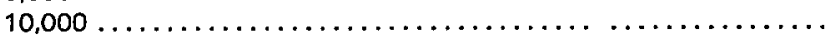 & 0.6 & 1.3 & 1.8 & 2.4 & 2.8 & 3.0 \\
\hline 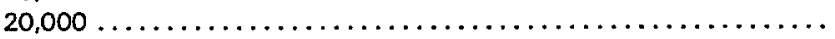 & 0.4 & 0.9 & 1.3 & 1.7 & 2.0 & 2.1 \\
\hline 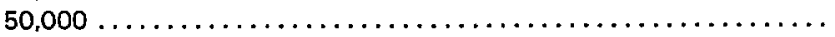 & 0.3 & 0.6 & 0.8 & 1.1 & 1.2 & 1.3 \\
\hline $100,000 \ldots \ldots \ldots \ldots \ldots \ldots \ldots \ldots \ldots \ldots \ldots \ldots \ldots \ldots \ldots \ldots \ldots \ldots \ldots$ & 0.2 & 0.4 & 0.6 & 0.8 & 0.9 & 1.0 \\
\hline 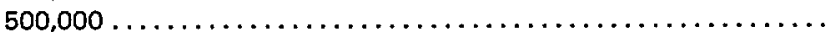 & 0.1 & 0.2 & 0.3 & 0.3 & 0.4 & 0.4 \\
\hline
\end{tabular}

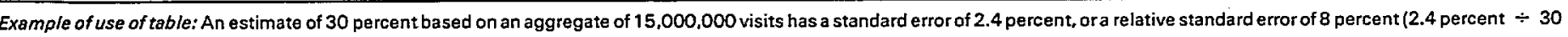
percent).

Table 111. Approximate relative standard errors of estimated number of drug mentions based on all physician specialties: NAMCS, 1980

\begin{tabular}{|c|c|}
\hline $\begin{array}{l}\text { Estimated number of drug mentions } \\
\text { in thousands }\end{array}$ & $\begin{array}{c}\text { Relative } \\
\text { standard } \\
\text { error in } \\
\text { percent }\end{array}$ \\
\hline 1,000 & 27.3 \\
\hline 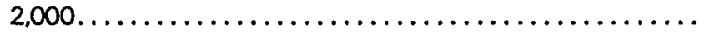 & $i 9.7$ \\
\hline 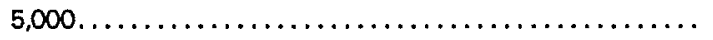 & 13.2 \\
\hline$\ldots \ldots \ldots \ldots \ldots$ & 10.1 \\
\hline$\ldots \ldots \ldots \ldots \ldots \ldots \ldots \ldots \ldots \ldots \ldots \ldots \ldots \ldots$ & 8.2 \\
\hline 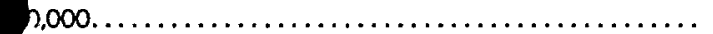 & 6.8 \\
\hline$\ldots \ldots \ldots, \ldots, \ldots, \ldots, \ldots, \ldots, \cdots$ & 6.2 \\
\hline 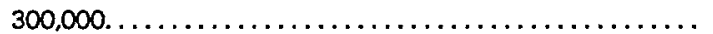 & 5.8 \\
\hline 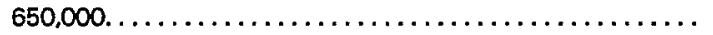 & 5.7 \\
\hline
\end{tabular}

Example of use of table: An aggregate estimate of $75,000,000$ drug mentions has a relative standard error of 6.5 percent, or a standard error of $4,875,000$ mentions (6.5 percent of $75,000,000)$. location for his ambulatory practice. Responsibility over tume for patient care and professional services rendered there generally resides with the individual physician rather than an institution.

A visit is a direct personal exchange between an ambulatory patient and a physician or a staff member working under the physician's supervision, for the purpose of seeking care and rendering health services.

Adrug mention is the physician's entry of a pharmaceutical agent ordered or provided-by any route of administration - for prevention, diagnosis, or treatment. Generic as well as brand-name drugs are included, as are nonprescription as well as prescription drugs. Along with all new drugs, the physician also records continued medications if the patient was specifically instructed during the visit to continue the medication.

Table IV. Approximate standard errors of percents of estimated numbers of drug mentions based on all physician specialties: NAMCS, 1980

Base of percent

(number of drug mentions in thousands)

\begin{tabular}{lllllll}
\multicolumn{6}{c}{ Estimated percent } \\
\hline 1 or99 & 5 or 95 & 10 or 90 & 20 or 80 & 30 or 70 & 50 \\
\hline
\end{tabular}

Standard error in percentage points

\begin{tabular}{|c|c|c|c|c|c|c|}
\hline 1,000 & 2.7 & 5.8 & 8.0 & 10.7 & 12.2 & 13.3 \\
\hline 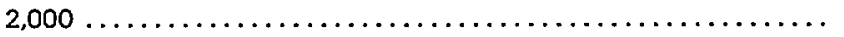 & 1.9 & 4.1 & 5.7 & 7.6 & 8.7 & 9.4 \\
\hline 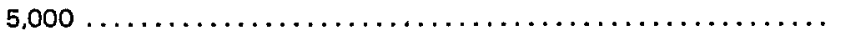 & 1.2 & 2.6 & 3.6 & 4.8 & 5.5 & 6.0 \\
\hline 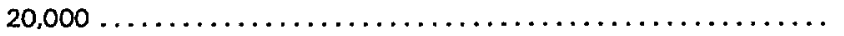 & 0.6 & 1.3 & 1.8 & 2.4 & 2.7 & 3.0 \\
\hline 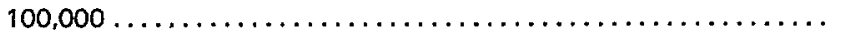 & 0.3 & 0.6 & 0.8 & 1.1 & 1.2 & 1.3 \\
\hline 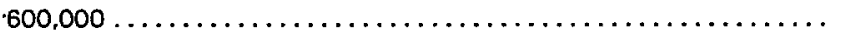 & 0.1 & 0.2 & 0.3 & 0.4 & 0.5 & 0.5 \\
\hline
\end{tabular}

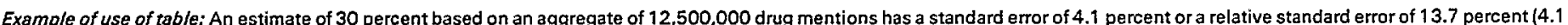
percent $\div 30$ percent). 


\section{Symbols}

- - Data not available

... Category not applicable

- Quantity zero

0.0 Quantity more than zero but less than 0.05

Z Quantity more than zero but less than $\mathbf{5 0 0}$ where numbers are rounded to thousands

* Figure does not meet standards of reliability or precision

\# $\quad$ Figure suppressed to comply with confidentiality requirements

\section{Recent Issues of Advance Data From Vital and Health Statistics}

No. 85. Summary Data From the National Inventory of Pharmacists: United States, 1978-79 (Issued: October 8, 1982)

No. 84. Blood Pressure Levels and Hypertension in Persons Ages 6-74 Years: United States, 1976-80 (Issued: October 8, 1982)

No. 83. Deliveries in Short-Stay Hospitals: United States, 1980 (Issued: October 8, 1982)
No. 82. Contraceptive Use Patterns, Prior Source, and Pregnancy History of Female Family Planning Patients: United States, 1980 (Issued: June 16, 1982)

No. 81. Drug Utilization in Office Practice by Age and Sex of the Patient: National Ambulatory Medical Care Survey, United States, 1980 (Issued: July 26, 1982)

SUGGESTED CITATION

National Center for Health Statistics, B. K. Cypress:

Drug utilization in office visits to primary care

physicians: National Ambulatory Medical Care Survey,

1980. Advance Data From Vital and Health Statistics,

No. 86. DHHS Pub. No. (PHS) 82-1250. Public

Health Service, Hyattsville, Md. October 8, 1982

\section{COPYRIGHT INFORMATION}

This report may be reprinted without further permission.

POSTAGE AND FEES PAID

U.S. DEPARTMENT OF HHS

HHS 396

AND HUMAN SERVICES

Public Health Service

National Center for Health Statistics

3700 East-West Highway

Hyattsville, Maryland 20782

THIRD CLASS

BULK RATE

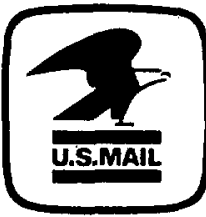

OFFICIAL BUSINESS

PENALTY FOR PRIVATE USE, $\$ 300$

To receive this publication regularly, contact the

National Center for Health Statistics by calling

301-436-NCHS 